\title{
Interior Characterization in Multiplanetary Systems: TRAPPIST-1
}

\author{
Caroline Dorn ${ }^{1}$, Klaus Mosegaard ${ }^{2}$, Simon L. Grimm ${ }^{1,3}$ (1) , and Yann Alibert ${ }^{3}$ (1) \\ ${ }^{1}$ University of Zurich, Institut of Computational Sciences, University of Zurich, Winterthurerstrasse 190, CH-8057, Zurich, Switzerland; cdorn@physik.uzh.ch \\ ${ }^{2}$ Niels-Bohr-Institute, Climate and Geophysics, Juliane Maries Vej 30, DK-2100 Copenhagen, Denmark \\ ${ }^{3}$ University of Bern, Center for Space and Habitability, Gesellschaftsstrasse 6, CH-3012, Bern, Switzerland \\ Received 2018 May 21; revised 2018 July 26; accepted 2018 August 4; published 2018 September 18
}

\begin{abstract}
Interior characterization traditionally relies on individual planetary properties, ignoring correlations between different planets of the same system. For multiplanetary systems, planetary data are generally correlated. This is because the differential masses and radii are better constrained than absolute planetary masses and radii. We explore such correlations and data specific to the multiplanetary system of TRAPPIST-1 and study their value for our understanding of planet interiors. Furthermore, we demonstrate that the rocky interior of planets in a multiplanetary system can be preferentially probed by studying the densest planet representing a rocky interior analog. Our methodology includes a Bayesian inference analysis that uses a Markov chain Monte Carlo scheme. Our interior estimates account for the anticipated variability in the compositions and layer thicknesses of core, mantle, water oceans, and ice layers, as well as a gas envelope. Our results show that (1) interior estimates significantly depend on available abundance proxies and (2) the importance of interdependent planetary data for interior characterization is comparable to changes in data precision by $30 \%$. For the interiors of TRAPPIST-1 planets, we find that possible water mass fractions generally range from $0 \%$ to $25 \%$. The lack of a clear trend of water budgets with orbital period or planet mass challenges possible formation scenarios. While our estimates change relatively little with data precision, they critically depend on data accuracy. If planetary masses varied within $\pm 24 \%$, interiors would be consistent with uniform $(\sim 7 \%)$ or an increasing water mass fractions with orbital period $(\sim 2 \%-12 \%)$.
\end{abstract}

Key words: methods: statistical - planets and satellites: composition - planets and satellites: individual (TRAPPIST-1) - planets and satellites: interiors - planets and satellites: oceans - planets and satellites: terrestrial planets

\section{Introduction}

Among all exoplanetary systems known today, the TRAPPIST-1 system harbors the largest number of Earth-sized exoplanets in a single system. It is a tightly packed system in which at least seven planets orbit an ultracool star (Gillon et al. 2017). The proximity of the planets to their central star and the low stellar mass and stellar luminosity imply temperate conditions for the planets, with equilibrium temperatures ranging from about 160 to $400 \mathrm{~K}$. Although characteristics of the planets remind us of Earth and Venus, the characteristics of star and system architecture seem very exotic compared to our solar system. How systems like TRAPPIST-1 formed and evolved over time is an open and fascinating question that has motivated plenty of exoplanetary studies.

Key to our understanding of the formation and evolution is the knowledge of the composition and structure of the planetary interiors. Our ability to characterize planetary interiors depends on available observations and prior information. Prior information is based on laboratory work and theoretical considerations and yields important constraints, e.g., on the anticipated range of possible interiors. Astrophysical observations provide, e.g., planetary mass, planetary radius, and orbital period.

For our study, the observational data on planetary radii and masses are taken from Delrez et al. (2018) and Grimm et al. (2018), respectively. The observed transit depths constrain ratios of planetary-to-stellar radii $R_{\mathrm{p}} / R_{\star}$ for each planet, while the transit timing variations (TTVs) constrain ratios of planetary-to-stellar masses $M_{\mathrm{p}} / M_{\star}$ from gravitational interactions among all seven planets and the star. The planet bulk densities are then calculated from $\rho_{\mathrm{p}}=\rho_{\star} M_{\mathrm{p}} / M_{\star} R_{\star}^{3} / R_{\mathrm{p}}^{3}$, given the photometrically well-constrained stellar density $\rho_{\star}$ (Seager $\&$ Mallen-Ornelas 2003). There are three important consequences that stem from this: (1) mass and radius for each planet are correlated, (2) planetary masses between the planets as derived by TTV are correlated, and (3) planetary radii between the planets are correlated. The correlation of the masses between all planets is a consequence of the planets being in a full resonance chain with each other. The correlation of planetary radii stems from the differential radii (or differential transit depths) being better constrained than the absolute radii because the latter include the uncertainty on stellar radii. Correlation of data can have significant influences on the range of inferred planetary interiors.

For an individual planet, the correlation of mass and radius has been taken into account for exoplanet interior characterizations (Weiss et al. 2016; Crida et al. 2018). However, the correlation of data between different planets of a single system has so far not been taken into account. In the present work, we develop a new resampling scheme that formally accounts for this interdependency of planetary data. We demonstrate how our ability of constraining interiors is affected by data interdependencies using the example of planetary masses. We generally believe that it is important to make thorough use of all available information (including the discussed data interdependencies) because observational data are few and expensive. Here we provide the tool to do so.

Previous characterization studies demonstrated the value of refractory rock-forming element abundances as constraints in addition to planetary mass and radius (Sotin et al. 2007; 
Dorn et al. 2015). Estimating photospheric abundances of this faint $(V=19)$ and cool $(\sim 2500 \mathrm{~K})$ host star is unfortunately still very challenging. Measured elemental abundances in the stellar photosphere may otherwise be used as abundance proxies for the rocky planetary interiors. Given a multiplanetary system, we can derive an abundance proxy based on the densest planet of the system, because its interior will be dominantly rocky. TRAPPIST-1e is the densest and probably a purely rocky planet, given its high bulk density. Thus, planet e may be seen as a rocky interior analog of all planets in the system. We analyze the possible range of elemental abundances for planet TRAPPIST-1e given only its mass and radius without abundance constraints. The obtained range of bulk abundances of TRAPPIST-1e is subsequently used as constraints for the other planets. Alternatively, we also investigate the abundance constraint that was suggested by Unterborn et al. (2017).

We note that preliminary tests including new observations for TRAPPIST-1 that were previously unavailable for the study of Grimm et al. (2018) indicate shifts in planet masses within $\pm 24 \%$ (Demory 2018). Also, Kane (2018) indicates changes in planetary radii on the order of $+2 \%$. Therefore, our results and those of Suissa \& Kipping (2018) and Unterborn et al. (2018) should be taken with care. We show how much such systematic shifts could affect our interior estimates (Section 5.1). Nonetheless, our paper demonstrates how interior characterization for multiplanetary systems has extraordinary advances compared to individual planets.

The structure of our study is as follows. We briefly review previous studies on possible interiors of the TRAPPIST-1 planets (Section 2). Then we describe our methodology that involves the new resampling scheme (Section 3). We demonstrate and discuss our results (Section 4) in light of possible formation and evolution paths of the planets (Section 5), and then we provide a summary (Section 6).

\section{Previous Studies}

Masses and radii of the planets as estimated by Gillon et al. (2017) suggest that all planets can have volatiles in either gas or water layers or can be rocky, while only planet f actually requires a significant layer of volatiles in order to explain its low bulk density.

Given the mass and radius estimates from Gillon et al. (2017) and Luger et al. (2017), Unterborn et al. (2017) estimated the water mass fraction to be more than $50 \%$ for planets $\mathrm{f}$ and $\mathrm{g}$, and on the order of $7 \%$ for planets $\mathrm{b}$ and $\mathrm{c}$. They concluded that the difference in water mass fraction is because of a difference in formation location relative to the ice line.

Since tidal interactions between the planets may affect the interiors, Barr et al. (2018) estimated the tidal heat fluxes on planets d, e, and f and estimated them to be 20 times higher than Earth's mean surface heat flow. They suggest that magma oceans could be maintained on planets $b$ and $c$.

Given the bulk densities, thick atmospheres may cover the planets. Such thick atmospheres could be realized by a few bars of hydrogen; however, atmospheric escape around TRAPPIST1 is strong enough to limit the lifetime of $\mathrm{H}_{2}$ atmospheres given the large EUV irradiation (Bolmont et al. 2016) or even considering cometary impacts (Kral et al. 2018). Indeed, transit spectroscopy for all planets shows no evidence for a cloud/ haze-free $\mathrm{H}_{2}$-dominated atmosphere (de Wit et al. 2016, 2018), except for planet $g$, for which data remain inconclusive. Thus, both atmospheric observations and theoretical considerations of atmospheric loss strongly suggest the presence of terrestrialtype atmospheres.

Turbet et al. (2018) highlight the synchronous rotation of the planets and study consequences for the diversity of climates on the planets. The nightside temperatures would allow atmospheric $\mathrm{CO}_{2}$ to condense out to form ice shields. For the outer planets $\mathrm{g}$ and $\mathrm{h}$, a global $\mathrm{CO}_{2}$ ice cover is possible. However, they also show that for planets $\mathrm{g}$ and $\mathrm{f}$, greenhouse warming could be efficient enough to allow liquid water.

Loss of water over the evolution of the planets has been investigated by Bourrier et al. (2017). They suggest that water loss could have been efficient enough to remove a few to several tens of Earth oceans over the planet's lifetime. Compared to estimates of possible water mass fractions $(7 \%-$ $50 \%$ ), this is very small (1 Earth ocean is $0.02 \%$ of Earth's total mass).

The most recent and more precise mass and density estimates from Grimm et al. (2018) provide new insights into the planet bulk compositions. They find that purely rocky interiors are likely for planets c and e, while planets b, d, f, g, and h require envelopes of volatiles with water mass fractions generally less than about $15 \% .{ }^{4}$ For the inner planets $b-d$, volatiles are likely in the form of an atmosphere given the high stellar irradiation. The outer planets $\mathrm{f}-\mathrm{h}$ have cold enough equilibrium temperatures that common volatile species $\mathrm{CO}_{2}$ and $\mathrm{H}_{2} \mathrm{O}$ are condensed out. Planet e has been recognized by different authors (e.g., Turbet et al. 2018; Grimm et al. 2018) to have the largest potential for Earth-like surface conditions.

Here we provide distributions of possible interiors using a Bayesian inference analysis while using all the information from the calculated mass and radius distributions as provided by Delrez et al. (2018) and Grimm et al. (2018). Furthermore, our physical interior model accounts for interiors of general structures and compositions that have been developed to describe the full range of super-Earths to mini-Neptunes. Also, we test different abundance proxies for refractory elements and account for the correlation of planetary data between different planets.

\section{Method}

The aim of this study is to calculate confidence regions of interior parameters that are linked to the formation and evolution of the planets. There are two sources of information that allow us to constrain interior parameters: observable data and prior information on the interior. Data $\boldsymbol{d}$ and interior parameters $\boldsymbol{m}$ are linked by the interior model that is incorporated in the operator $g(\cdot)$, such that $\boldsymbol{d}=g(\boldsymbol{m})$. In our case, the operator $g(\cdot)$ is not invertible. Therefore, we need to infer the possible realizations of $\boldsymbol{m}$ given the data $\boldsymbol{d}$ using inference methods. Here we use a Bayesian inference analysis based on a Markov chain Monte Carlo (MCMC) scheme by Dorn et al. (2015). This scheme characterizes every planet individually. However, there is a clear correlation of planetary data between the planets. We account for this correlation by introducing a novel resampling scheme. In the following, we discuss the different aspects of the method.

\footnotetext{
4 In the published version their Figure 10 correctly compares differen interiors with the planetary data. However, in the text the erroneously stated $5 \%$ should be $15 \%$ and $15 \%$ should be $35 \%$.
} 


\subsection{Interior Model}

The interior model $g(\cdot)$ assumes planets to be composed of a pure iron core, a silicate mantle comprising the oxides $\mathrm{Na}_{2} \mathrm{O}-\mathrm{CaO}-\mathrm{FeO}-\mathrm{MgO}-\mathrm{Al}_{2} \mathrm{O}_{3}-\mathrm{SiO}_{2}$, a pure water layer, and an isothermal gas layer. Those model parameters $\boldsymbol{m}$ that determine the rocky and the water layers are $r_{\text {core }}, r_{\text {core+mantle }}$, $\mathrm{Fe} / \mathrm{Si}_{\text {mantle }}, \mathrm{Mg} / \mathrm{Si}_{\text {mantle }}$, and $m_{\text {water }}$.

The structural model for the interior uses self-consistent thermodynamics for core, mantle, high-pressure ice, and water ocean. For the core density profile, we use the equation-of-state (EOS) fit of iron in the hcp (hexagonal close-packed) structure provided by Bouchet et al. (2013) on ab initio molecular dynamics simulations. For the silicate mantle, we compute equilibrium mineralogy and density as a function of pressure, temperature, and bulk composition by minimizing Gibbs free energy (Connolly 2009). For the water layers, we follow Vazan et al. (2013) using a quotidian equation of state (QEOS), and above 44.3 GPa, we use the tabulated EOS from Seager et al. (2007) that is derived from density functional theory (DFT) simulations. Depending on pressure and temperature, the water can be in solid, liquid, or supercritical phase. Water vapor is excluded, since we impose the condition that if water is present, then there must be a gas layer on top that imposes a pressure at least as high as the vapor pressure of water. We assume an adiabatic temperature profile within each layer of core, mantle, and solid water. The surface temperature of the water layer is set equal to the temperature of the bottom of the gas layer. Also, temperature is assumed to be continuous across other layer boundaries.

For the gas layer, we test two different models: an isothermal model (model I) and a fully adiabatic model (model II). Model I assumes a thin, isothermal atmosphere in hydrostatic equilibrium and ideal gas behavior, which is calculated using the scale height model. Those model parameters of $\boldsymbol{m}$ that parameterize the gas layer in model I and that we aim to constrain are the pressure at the bottom of the gas layer $P_{\text {batm }}$, the mean molecular weight $\mu$, and the mean temperature (parameterized by $\alpha$; see below). The thickness of the opaque gas layer $d_{\text {atm }}$ in model I is given by

$$
d_{\text {atm }}=H \ln \frac{P_{\text {batm }}}{P_{\text {out }}},
$$

where the amount of opaque scale heights $H$ is determined by the ratio of $P_{\text {batm }}$ and $P_{\text {out }} . P_{\text {out }}$ is the pressure level at the optical photosphere for a transit geometry that we fix to 20 mbar (Fortney et al. 2007). The scale height $H$ is the increase in altitude for which the pressure drops by a factor of $e$ and can be expressed by

$$
H=\frac{T_{\text {atm }} R^{*}}{g_{\text {batm }} \mu},
$$

where $g_{\text {batm }}$ and $T_{\text {atm }}$ are gravity at the bottom of the atmosphere and mean atmospheric temperature, respectively. $R^{*}$ is the universal gas constant $\left(8.3144598 \mathrm{~J} \mathrm{~mol}^{-1} \mathrm{~K}^{-1}\right)$, and $\mu$ is the mean molecular weight. The mass of the atmosphere $m_{\text {atm }}$ is directly related to the pressure $P_{\text {batm }}$ as

$$
m_{\mathrm{atm}}=4 \pi P_{\text {batm }} \frac{r_{\text {batm }}^{2}}{g_{\text {batm }}},
$$

where $r_{\text {batm }}$ is the radius at the bottom of the atmosphere.
Table 1

Prior Ranges

\begin{tabular}{lll}
\hline \hline Parameter & Prior Range & Distribution \\
\hline$r_{\text {core }}$ & $(0.01-1) r_{\text {mantle }}$ & Uniform in $r_{\text {core }}^{3}$ \\
$\mathrm{Fe} / \mathrm{Si}_{\text {mantle }}$ & $0-\mathrm{Fe} / \mathrm{Si}_{\text {star }}$ & Uniform \\
$\mathrm{Mg} / \mathrm{Si}_{\text {mantle }}$ & $\mathrm{Mg} / \mathrm{Si}_{\text {star }}$ & Gaussian \\
$r_{\text {core }+ \text { mantle }}$ & $(0.01-1) R$ & Uniform in $r_{\text {mantle }}^{3}$ \\
$m_{\text {water }}$ & $0-0.98 M$ & Uniform \\
$P_{\text {batm }}$ & 20 mbar $-P_{\text {batm,max }}$ & Uniform in $\ln \left(P_{\text {batm }}\right)$ \\
$\alpha($ model I) & $0.5-\alpha_{\text {max }}$ & Uniform \\
$\alpha($ model II $)$ & $0.5-1$. & Uniform \\
$\mu$ & $2.3-50.0$ & Uniform in $1 / \mu$ \\
$n$ (model II) & $5-6$ & Uniform \\
\hline
\end{tabular}

The atmosphere's constant temperature is defined as

$$
T_{\mathrm{atm}}=\alpha T_{\mathrm{star}} \sqrt{\frac{R_{\mathrm{star}}}{2 a}},
$$

where $R_{\text {star }}$ and $T_{\text {star }}$ are the radius and effective temperature of the host star, respectively, and $a$ is the semimajor axis. The factor $\alpha$ accounts for possible cooling and heating of the atmosphere and can vary between 0.5 and $\alpha_{\max }$. The upper bound $\alpha_{\max }$ is because there is a physical limit to the amount of warming by greenhouse gases. We approximate $\alpha_{\max }$ for a moist (water-saturated) atmosphere (see Appendix A; Dorn et al. 2017).

The assumption of a constant mean temperature in model I might affect the interior predictions. In order to test how sensitive our results are to this assumption, we propose a second atmosphere model (model II) that assumes a convective adiabat. For model II, the temperature at the bottom of the gas layer, $T_{\text {batm }}$, is calculated as

$$
T_{\text {batm }}=T_{\text {out }}\left(\frac{P_{\text {batm }}}{P_{\text {out }}}\right)^{\kappa},
$$

where $T_{\text {out }}$ is calculated with Equation (4), while restricting $\alpha$ to values between 0.5 and 1 , which is equivalent to a range of albedos from 0 to 0.94 . The exponent $\kappa$ is equal to $2 /(2+n)$, with $n$ being the number of degrees of freedom (which we vary randomly between 5 and 6 for diatomic or triatomic gases, thus neglecting vibrational modes). Following a convective adiabat, the thickness of the opaque atmosphere is set to

$$
d_{\mathrm{atm}}=\frac{c_{\mathrm{p}}}{g_{\text {batm }}}\left(T_{\mathrm{batm}}-T_{\mathrm{atm}}\right),
$$

where the heat capacity is $c_{\mathrm{p}}=(n / 2+1) R^{*} / \mu$.

In summary, the entire interiors are parameterized by the full set of interior parameters $\boldsymbol{m}$ : $r_{\text {core }}, r_{\text {core }+ \text { mantle }}, \mathrm{Fe} / \mathrm{Si}_{\text {mantle }}$, $\mathrm{Mg} / \mathrm{Si}_{\text {mantle }}, m_{\text {water }}, P_{\text {batm }}, \alpha, \mu$, and for model II also $n$. We refer to Dorn et al. (2017) for more details on the interior structure model.

\subsection{Prior Information}

The prior distributions of the interior parameters are listed in Table 1. The priors are chosen conservatively. The cubic uniform priors on $r_{\text {core }}$ and $r_{\text {core }+ \text { mantle }}$ reflect equal weighing of masses for both core and mantle. Prior bounds on $\mathrm{Fe} / \mathrm{Si}_{\text {mantle }}$ and $\mathrm{Mg} / \mathrm{Si}_{\text {mantle }}$ are determined by the abundance proxies. Since iron is distributed between core and mantle, the 


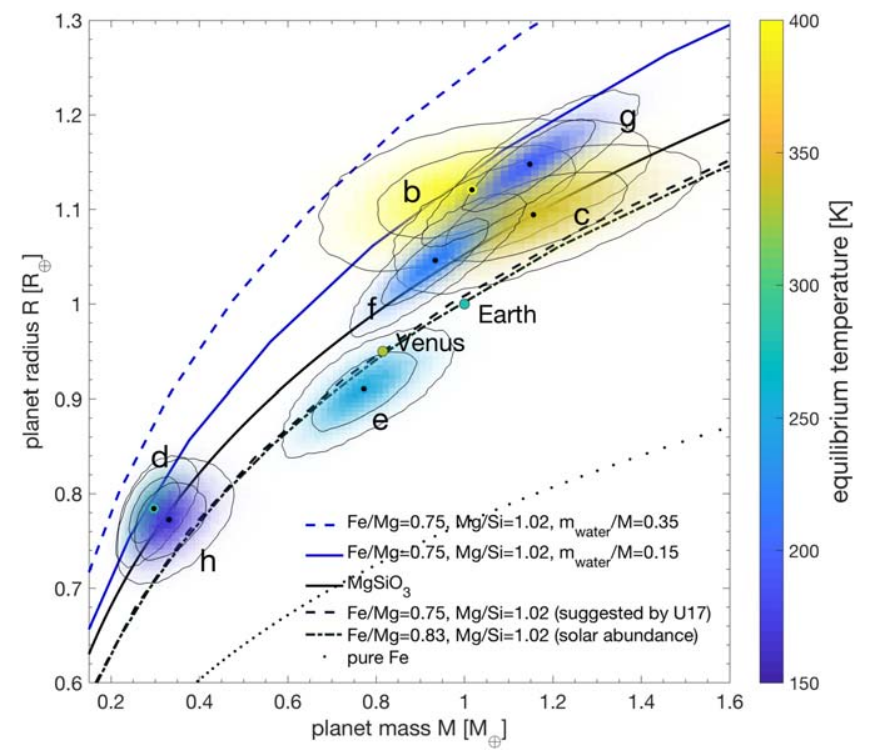

Figure 1. Mass-radius plot showing TRAPPIST-1 planets, Earth and Venus. The plot is adapted from Grimm et al. (2018). U17 refers to Unterborn et al. (2017).

abundance constraint only sets an upper bound on $\mathrm{Fe} / \mathrm{Si}_{\text {mantle }}$, thus making iron-free mantles possible. For the atmospheric models I and II, a ln-uniform prior is set for $P_{\mathrm{batm}} . P_{\mathrm{batm}, \max }$ is estimated by Equation (24) from Ginzburg et al. (2016), who investigated super-Earth atmospheres. A uniform prior in $\mu$ equally favors various dominating species (e.g., $\mathrm{N}_{2}, \mathrm{CO}_{2}$, $\mathrm{H}_{2} \mathrm{O}$ ), which seems appropriate for anticipated terrestrial-like atmospheres. The factor $\alpha$ incorporates possible cooling (model I and II) and heating (model I) of the atmosphere; it can vary linearly between 0.5 and $\alpha_{\max }$ for the isothermal model I and between 0.5 and 1 for the adiabatic model II. The upper bound $\alpha_{\max }$ is a physical limit to the amount of warming by greenhouse gases and is described in Appendix A. The lower bound of 0.5 is equivalent to an albedo of 0.94 , which is similar to the highest albedo estimate among solar system bodies (0.96 for Eris; Sicardy et al. 2011).

\subsection{Data}

The data that we use to characterize the interiors of the planets comprise planetary mass and radius, stellar irradiation, and constraints on refractory element abundances. Planetary radii are taken from Delrez et al. (2018), masses and semimajor axes are taken from Grimm et al. (2018), and stellar radius and luminosity are given by Gillon et al. (2017). These data are based on several hundred planet transits that were observed between 2015 September and 2017 March. In addition to the transits presented in earlier studies (de Wit et al. 2016; Gillon et al. 2016, 2017; Luger et al. 2017), Grimm et al. (2018) included additional observations from Spitzer Space Telescope, Kepler, and $K 2$.

For TRAPPIST-1, there is a correlation between planetary radius and mass for the individual planets, as well as a correlation between all seven planetary masses and between all seven planetary radii. In order to better distinguish between the two kinds of correlation, we will use the following nomenclature. Unless otherwise mentioned, the terms correlated data and uncorrelated data refer to the correlation between mass and radius of individual planets, while the terms dependent and
Table 2

Masses, Radii, and Their Correlation Coefficients $c_{\mathrm{m}, \mathrm{r}}$ of All Seven Planets

\begin{tabular}{lccccccc}
\hline \hline Planet & $m\left(M_{\oplus}\right)$ & $-\sigma$ & $+\sigma$ & $R\left(R_{\oplus}\right)$ & $-\sigma$ & $+\sigma$ & $c_{\mathrm{m}, \mathrm{r}}$ \\
\hline $\mathrm{b}$ & 1.017 & 0.143 & 0.154 & 1.121 & 0.032 & 0.031 & 0.502 \\
$\mathrm{c}$ & 1.156 & 0.131 & 0.142 & 1.095 & 0.031 & 0.030 & 0.624 \\
$\mathrm{~d}$ & 0.297 & 0.035 & 0.039 & 0.784 & 0.023 & 0.023 & 0.569 \\
$\mathrm{e}$ & 0.772 & 0.075 & 0.079 & 0.910 & 0.027 & 0.026 & 0.708 \\
$\mathrm{f}$ & 0.934 & 0.078 & 0.080 & 1.046 & 0.030 & 0.029 & 0.855 \\
$\mathrm{~g}$ & 1.148 & 0.095 & 0.098 & 1.148 & 0.033 & 0.032 & 0.863 \\
$\mathrm{~h}$ & 0.331 & 0.049 & 0.056 & 0.773 & 0.027 & 0.026 & 0.386 \\
\hline
\end{tabular}

independent refer to the correlation between planetary data of the different planets. Planetary masses and radii are shown in Figure 1 and listed in Table 2, where we also list the corresponding correlation $c_{\mathrm{m}, \mathrm{r}}$. Figure 2 shows the interdependency of planetary masses as derived from Grimm et al. (2018).

For the constraints on refractory element abundances we have compiled two different scenarios. In the first scenario (U), we use the bulk abundance constraints as suggested by Unterborn et al. (2017), who analyzed F-G-K stars of similar metallicity to TRAPPIST-1 and estimated their statistical median to $\mathrm{Fe} / \mathrm{Mg}=1.72 \pm 0.46$ in mass ratios, while fixing the $\mathrm{Mg} / \mathrm{Si}$ mass ratio to 0.87 . Here we adopted these estimates: $\mathrm{Fe} / \mathrm{Si}=1.49 \pm 0.4, \mathrm{Mg} / \mathrm{Si}=0.89 \pm 0.3$. For comparison, the solar mass ratio estimates are $\mathrm{Fe} / \mathrm{Si}=1.69$ and $\mathrm{Mg} / \mathrm{Si}=0.89$ (Lodders et al. 2009). In a second scenario (A), we do not impose any constraints on bulk abundances that are based on stellar estimates. Instead, we analyze the possible range of refractory elements for the densest planet, TRAPPIST1e, while only using its data of mass, radius, and stellar irradiation. The estimated ranges of $\mathrm{Mg} / \mathrm{Si}_{\mathrm{T} 1 \mathrm{e}}$ and $\mathrm{Fe} / \mathrm{Si}_{\mathrm{T} 1 \mathrm{e}}$ are subsequently used as input constraints under the premise that planet $\mathrm{e}$ is analogous in its relative refractory element budget to the rocky interiors of all planets. This excludes a priori the possibility of planet e being a Mercury-type planet.

In total, we discuss five different data scenarios, labeled $\mathrm{U}, \mathrm{A}$, UCM, UHM, and UHMR, which we analyze in detail. All data scenarios are summarized in Table 3. Scenarios $U$ and A differ in terms of the abundance constraints used; in scenario UCM, we additionally account for the fact that planetary masses of different planets depend on each other; for UHM and UHMR, we use hypothetical reduced uncertainties on planetary masses and radii. All scenarios help to determine the value of the different data with respect to making interior predictions.

\subsection{Interior Characterization Scheme}

Our characterization scheme involves two main steps. The first step comprises the interior characterization of individual planets $\mathrm{b}-\mathrm{h}$ using the generalized Bayesian inference scheme of Dorn et al. (2017). The outputs are posterior distributions for each of the planets that do not depend on each other. In a second step, we account for the interdependency of the different planetary data. In order to do so, we have developed a resampling scheme that yields posterior distributions for each of the planets that depend on each other. Thereby, we take all available information of the mass-radius data into account.

In the following, we first introduce the formulation of Tarantola \& Valette (1982) on inference problems, and then we describe each of the two main steps. Our scheme is summarized in Figure 3. 


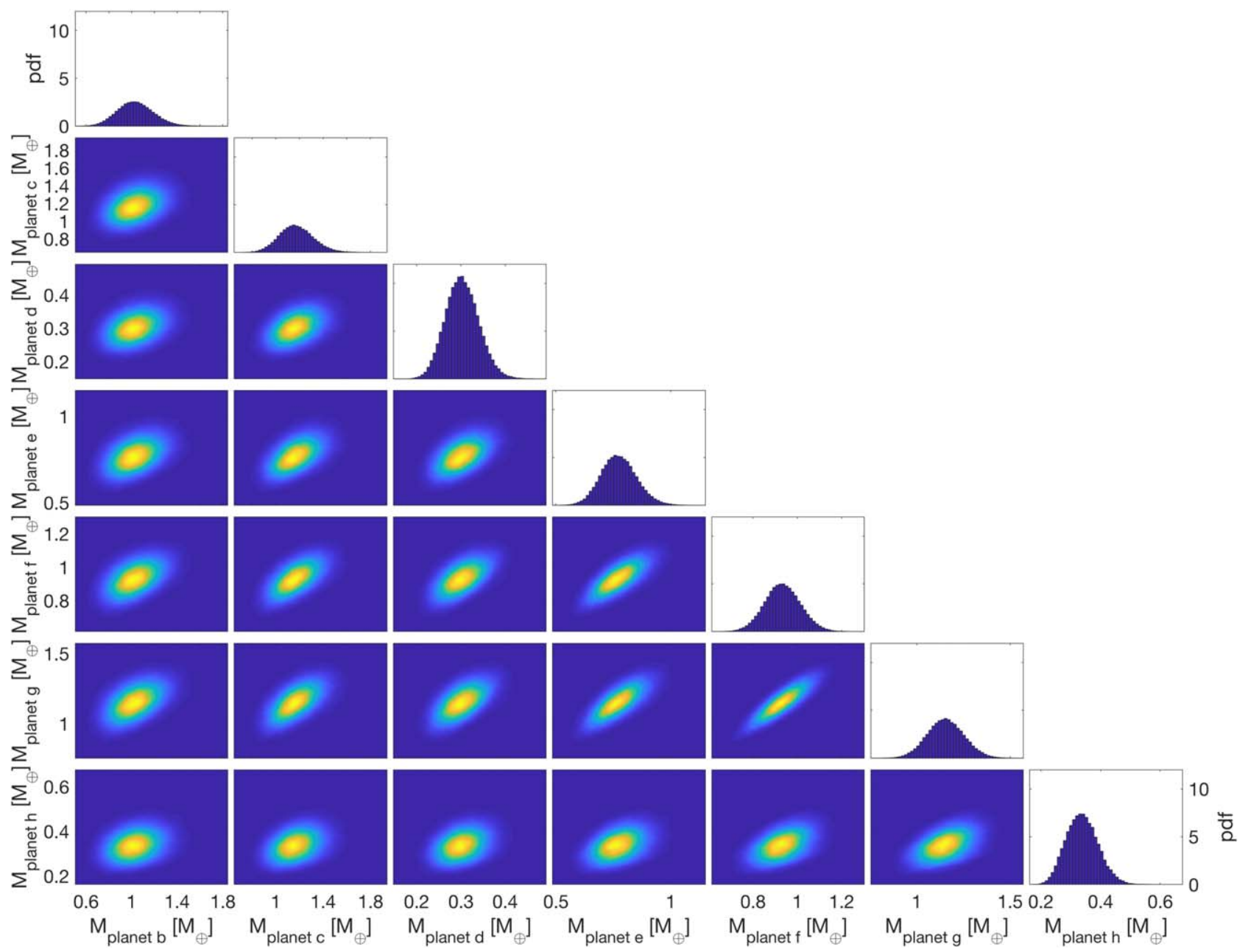

Figure 2. 2D marginal data distributions of planetary masses extracted from Figure 6 of Grimm et al. (2018).

\subsubsection{Bayes's Theorem and the Tarantola-Valette Formulation}

Formally, the posterior distribution based on Bayes's theorem for a fixed model parameterization and conditional on data is given by

$$
p(\boldsymbol{m} \mid \boldsymbol{d})=\frac{p(\boldsymbol{m}) L(\boldsymbol{m} \mid \boldsymbol{d})}{p(\boldsymbol{d})},
$$

where $p(\boldsymbol{m})$ represents prior information on model parameter $\boldsymbol{m}, L(\boldsymbol{m} \mid \boldsymbol{d})$ is the likelihood function, and $p(\boldsymbol{d})$ signifies the evidence and is here a normalizing constant. The likelihood function is defined as

$$
\begin{aligned}
L(\boldsymbol{m} \mid \boldsymbol{d})= & \frac{1}{(2 \pi)^{N / 2}(\operatorname{det} \Sigma)^{1 / 2}} \\
& \times \exp \left(-\frac{1}{2}(g(\boldsymbol{m})-\boldsymbol{d})^{T} \Sigma^{-1}(g(\boldsymbol{m})-\boldsymbol{d})\right),
\end{aligned}
$$

where $\Sigma$ is the data covariance matrix and $\operatorname{det}(\Sigma)$ denotes the determinant of $\Sigma$. For uncorrelated data errors, $\Sigma$ is a diagonal matrix. Here the correlation between planetary mass and radius is prescribed by the off-diagonal entries of $\Sigma$.

There is a different formulation of inference problems by Tarantola \& Valette (1982) that we will use in the following. In this formulation, the posterior $p(\boldsymbol{d}, \boldsymbol{m})$ is expressed in the joint space of data and model parameters $(\mathcal{D}$ and $\mathcal{M})$ as

$$
p(\boldsymbol{d}, \boldsymbol{m}) \equiv(\rho \wedge \theta)(\boldsymbol{d}, \boldsymbol{m})=\frac{\rho(\boldsymbol{d}, \boldsymbol{m}) \theta(\boldsymbol{d}, \boldsymbol{m})}{z(\boldsymbol{d}, \boldsymbol{m})},
$$

where the prior $\rho$, the forward model density $\theta$, and the null information density $z$ are also defined in the joint space $\mathcal{D} \times \mathcal{M}$. The null information is a normalizing constant. The posterior of the model parameters evaluated in the model space is then

$$
p_{m}(\boldsymbol{m})=\int_{\mathcal{D}} p(\boldsymbol{d}, \boldsymbol{m}) d \boldsymbol{d} .
$$

In Appendix B, we show that the Tarantola-Valette formulation is equivalent to Bayes's theorem under simplified conditions.

\subsubsection{Bayesian Inference of Individual Planets}

We use the probabilistic inference analysis of Dorn et al. (2017), who employ an MCMC. This method is used for every planet individually, and it computes a posterior probability density function (pdf) for each interior parameter $\boldsymbol{m}$ from data $\boldsymbol{d}$ and prior information. It is important to note that the data $\boldsymbol{d}$ used for each planet characterization are the marginalized data for each planet, such that data priors $\rho(\boldsymbol{d}, \boldsymbol{m})$ are independent. Thus, the obtained posteriors are independent of 
Table 3

Data Scenarios for Interior Characterization

\begin{tabular}{|c|c|c|c|c|c|c|}
\hline Considered Data & $\mathrm{N}$ & $\mathrm{U}$ & A & $\mathrm{UCM}$ & UHM & $\overline{\mathrm{UHMR}}$ \\
\hline Correlated $M_{\mathrm{p}}$ and $R_{\mathrm{p}}$ & $\checkmark$ & $\checkmark$ & $\checkmark$ & $\checkmark$ & $\checkmark$ & $\checkmark$ \\
\hline Stellar irradiation & $\checkmark$ & $\checkmark$ & $\checkmark$ & $\checkmark$ & $\checkmark$ & $\checkmark$ \\
\hline Interdependency of planetary masses $M_{\mathrm{p}}$ & $\times$ & $\times$ & $\times$ & $\checkmark$ & $\times$ & $\times$ \\
\hline Interdependency of planetary masses $M_{\mathrm{p}}$ excl. planet e & $\times$ & $\times$ & $\times$ & $\times$ & $\times$ & $\times$ \\
\hline Bulk abundances based on Unterborn et al. (2017) & $\times$ & $\checkmark$ & $\times$ & $\checkmark$ & $\checkmark$ & $\checkmark$ \\
\hline Bulk abundances based on TRAPPIST-1e & $x$ & $x$ & $\checkmark$ & $\times$ & $\times$ & $x$ \\
\hline Hypothetical reduced precision in $M_{\mathrm{p}}$ & $\times$ & $\times$ & $\times$ & $\times$ & $\times$ & $\checkmark$ \\
\hline Hypothetical reduced precision in $R_{\mathrm{p}}$ & $\times$ & $\times$ & $\times$ & $\times$ & $\checkmark$ & $\checkmark$ \\
\hline
\end{tabular}

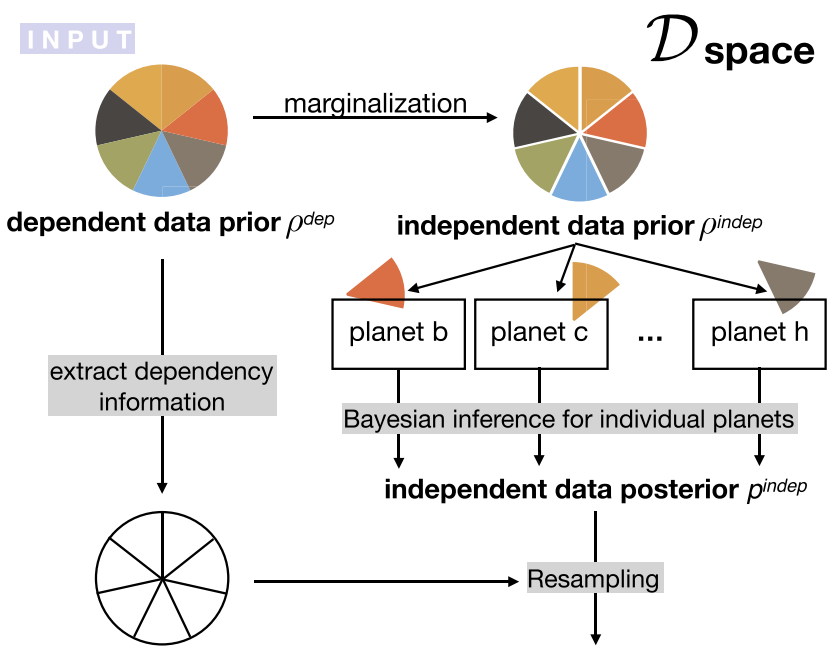

dependent data posterior $p^{d e p}$

Figure 3. Schematic of interior characterization scheme represented in the data space $\mathcal{D}$. Dependent data refers to the data that contain the interdependency of different planetary data. Independent data refers to the data of each planet that do not depend on other planets.

each other. The information on interdependency of data from different planets is ignored at this step and instead taken into account by our developed resampling scheme. The Bayesian inference analysis, in essence, explores the model space by sampling a huge number of model realizations $\boldsymbol{m}$ that are distributed according to the posterior distribution $p(\boldsymbol{d}, \boldsymbol{m})$. This is achieved within an iterative scheme where the ratio of likelihoods (Equation (8)) between subsequent sampled models is used as a criterion to accept or reject model realizations. The likelihood in probabilistic terms is a measure of how well a model realization fits the observed data. Here the posterior information is gathered from a large number of sampled models $\left(\sim 10^{6}\right)$. We refer to Dorn et al. (2015) for further details on the inference analysis.

\subsubsection{Resampling Scheme to Account for the Interdependency of Data from Different Planets}

The above-described Bayesian inference analysis yields posterior samples in the model and data space $(\mathcal{M}$ and $\mathcal{D})$, $p(\boldsymbol{d}, \boldsymbol{m})$. Note that the posterior data $p_{d}(\boldsymbol{d})$ can be different from the prior data $\rho_{d}(\boldsymbol{d})$. The data prior is the distribution of the noise-contaminated data, which is inherited by the given distribution of observational uncertainties. The posterior data are recomputed data, taking into account constraints from the model $\boldsymbol{m}$. Prior data and posterior data are therefore not

necessarily identical. The aim of our resampling is to preserve the differences between posterior and prior (which is added by model $\boldsymbol{m}$ ), while adding the information of the interdependency of prior data but not the prior data themselves (since these have been already used in the Bayesian inference).

The posteriors $p(\boldsymbol{d}, \boldsymbol{m})$ are obtained for each planet individually. Consequently, the obtained data posteriors for a specific planet evaluated in $\mathcal{D}, p_{d}(\boldsymbol{d})$, do not depend on the posteriors of other planets, but they are independent. The interdependency of planetary masses as described by Grimm et al. (2018) quantifies the dependencies of data priors between the planets. Formally, we can say that $\rho_{d_{i j}}\left(\boldsymbol{d}_{i j}\right) \neq \rho_{d_{i}}\left(\boldsymbol{d}_{i}\right) \rho_{d_{j}}\left(\boldsymbol{d}_{j}\right)$, where $i, j$ are generic planet indices $i, j \in\{b, c, d, e, f, g, h\}$. In the case in which the individual data priors are independent from each other, we can write the data posteriors as

$$
p_{d_{i j}}^{\text {indep }}(\boldsymbol{d})=L_{m_{i}}\left(\boldsymbol{d}_{i}\right) L_{m_{j}}\left(\boldsymbol{d}_{j}\right) \rho_{d_{i}}\left(\boldsymbol{d}_{i}\right) \rho_{d_{j}}\left(\boldsymbol{d}_{j}\right),
$$

while for dependent data priors the posteriors are

$$
\begin{aligned}
p_{d_{i j}}^{\mathrm{dep}}(\boldsymbol{d}) & =L_{m_{i j}}\left(\boldsymbol{d}_{i}, \boldsymbol{d}_{j}\right) \rho_{d_{i j}}\left(\boldsymbol{d}_{i}, \boldsymbol{d}_{j}\right) \\
& =L_{m_{i}}\left(\boldsymbol{d}_{i}\right) L_{m_{j}}\left(\boldsymbol{d}_{j}\right) \rho_{d_{i j}}\left(\boldsymbol{d}_{i}, \boldsymbol{d}_{j}\right) .
\end{aligned}
$$

$$
\begin{aligned}
& \text { If } L_{m_{i j}}\left(\boldsymbol{d}_{i}, \boldsymbol{d}_{j}\right)=L_{m_{i}}\left(\boldsymbol{d}_{i}\right) L_{m_{j}}\left(\boldsymbol{d}_{j}\right) \text {, then } \\
& \qquad p_{d_{i j}}^{\text {dep }}\left(\boldsymbol{d}_{i j}\right)=p_{d_{i j}}^{\text {indep }}\left(\boldsymbol{d}_{i j}\right) \frac{\rho_{d_{i j}}\left(\boldsymbol{d}_{i}, \boldsymbol{d}_{j}\right)}{\rho_{d_{i}}\left(\boldsymbol{d}_{i}\right) \rho_{d_{j}}\left(\boldsymbol{d}_{j}\right)} .
\end{aligned}
$$

The condition that $L_{m_{i j}}\left(\boldsymbol{d}_{i}, \boldsymbol{d}_{j}\right)=L_{m_{i}}\left(\boldsymbol{d}_{i}\right) L_{m_{j}}\left(\boldsymbol{d}_{j}\right)$ can be understood from the following. $L(\boldsymbol{d})$ is a measure of the likelihood of the specific data set $\boldsymbol{d}$, based solely on the prior information about the models $\boldsymbol{m}=g^{-1}(\boldsymbol{d})$ that map into $\boldsymbol{d}$. If $g$ is bijective (the inverse problem has a unique best-fitting solution), $L_{m}(\boldsymbol{d})=\rho_{m}\left(g^{-1}(\boldsymbol{d})\right)$. If the problem is nonunique, the right-hand side of this equation expresses an integral/sum over all models that map into $\boldsymbol{d}$. For simplicity, consider a case where our data consist of two (possibly dependent) parts, $\boldsymbol{d}_{1}$ and $\boldsymbol{d}_{2}$. In this case, the data likelihood can be expressed as

$$
L_{m}(\boldsymbol{d})=L_{m}\left(\boldsymbol{d}_{1}, \boldsymbol{d}_{2}\right)=\rho_{m_{1}, m_{2}}\left(g^{-1}\left(\boldsymbol{d}_{1}, \boldsymbol{d}_{2}\right)\right) .
$$

However, when $\boldsymbol{m}_{1}$ and $\boldsymbol{m}_{2}$ are a priori (statistically) independent and $\boldsymbol{m}_{i}$ is functionally related only to $\boldsymbol{d}_{i}$ (for $i=1,2$ ), we get

$$
\begin{gathered}
\rho_{m_{1}, m_{2}}\left(g^{-1}\left(\boldsymbol{d}_{1}, \boldsymbol{d}_{2}\right)\right)=\rho_{m_{1}}\left(g^{-1}\left(\boldsymbol{d}_{1}, \boldsymbol{d}_{2}\right)\right) \cdot \rho_{m_{2}}\left(g^{-1}\left(\boldsymbol{d}_{1}, \boldsymbol{d}_{2}\right)\right) \\
=\rho_{m_{1}}\left(g^{-1}\left(\boldsymbol{d}_{1}\right)\right) \cdot \rho_{m_{2}}\left(g^{-1}\left(\boldsymbol{d}_{2}\right)\right)
\end{gathered}
$$

leading to

$$
L_{m}\left(\boldsymbol{d}_{1}, \boldsymbol{d}_{2}\right)=L_{m_{1}}\left(\boldsymbol{d}_{1}\right) \cdot L_{m_{2}}\left(\boldsymbol{d}_{2}\right) .
$$

So much for the theory. 
In practice, the resampling works as follows. The inference analysis of individual planets yields independent data posteriors $p_{d_{i j}}^{\text {(indep) }}\left(\boldsymbol{d}_{i}, \boldsymbol{d}_{j}\right)=p_{d_{i}}\left(\boldsymbol{d}_{i}\right) p_{d_{j}}\left(\boldsymbol{d}_{j}\right)$. For a total of $K$ samples, we have $d_{i}^{1}, d_{i}^{2}, \ldots, d_{i}^{K}$ from $p_{d_{i}}$ and $d_{j}^{1}, d_{j}^{2}, \ldots, d_{j}^{K}$ from $p_{d_{j}}$. Thus, any pair (e.g., $\left.\left(d_{i}^{1}, d_{j}^{1}\right),\left(d_{i}^{2}, d_{j}^{2}\right), \ldots\left(d_{i}^{K}, d_{j}^{K}\right)\right)$ is a sample from $p_{d_{i}}\left(\boldsymbol{d}_{i}\right) p_{d_{j}}\left(\boldsymbol{d}_{j}\right)$. We shall resample from $p_{d_{i}}\left(\boldsymbol{d}_{i}\right) p_{d_{j}}\left(\boldsymbol{d}_{j}\right)$ such that the new samples are drawn from $p_{d_{i j}}^{\text {dep }}\left(\boldsymbol{d}_{i}, \boldsymbol{d}_{j}\right)$. In order to do so, we calculate a factor $Q$ that signifies a maximum difference between the data priors of the data-independent and data-dependent cases:

$$
Q=\max _{k}\left(\rho_{d_{i j}}^{\mathrm{dep}}\left(\boldsymbol{d}_{i}^{k}, \boldsymbol{d}_{j}^{k}\right) / \rho_{d_{i}}\left(\boldsymbol{d}_{i}^{k}\right) \rho_{d_{j}}\left(\boldsymbol{d}_{j}^{k}\right)\right) .
$$

For $k=1, \ldots, K$,

1. we generate a random number $w \in[0,1]$;

2. if $\quad w<\rho_{d_{i j}}^{\text {dep }}\left(\boldsymbol{d}_{i}^{k}, \boldsymbol{d}_{j}^{k}\right) /\left(Q \times \rho_{d_{i}}\left(\boldsymbol{d}_{i}^{k}\right) \rho_{d_{j}}\left(\boldsymbol{d}_{j}^{k}\right)\right), \quad$ then $\left(\boldsymbol{d}_{i}^{k}, \boldsymbol{d}_{j}^{k}\right)=\left(\boldsymbol{d}_{i}^{k}, \boldsymbol{d}_{j}^{k}\right)^{\mathrm{dep}}$; otherwise, discard the sample.

Thereby, we obtain samples $\left(\boldsymbol{d}_{i}^{k}, \boldsymbol{d}_{j}^{k}\right)^{\text {dep }}$ that represent the data posterior $p_{d_{i j}}^{\mathrm{dep}}\left(\boldsymbol{d}_{i}, \boldsymbol{d}_{j}\right)$. Any interior model pairs $\left(\boldsymbol{m}_{i}^{k}, \boldsymbol{m}_{j}^{k}\right)^{\mathrm{dep}}$ that generated $\left(\boldsymbol{d}_{i}^{k}, \boldsymbol{d}_{j}^{k}\right)^{\mathrm{dep}}$ are samples from the model posterior $p_{m_{i j}}^{\mathrm{dep}}\left(\boldsymbol{m}_{i}, \boldsymbol{m}_{j}\right)$.

In other words, we can understand the resampling as follows. We randomly combine samples from the analysis of individual planets $i$ and $j$, which represents a set of paired samples $\left(d_{i}^{k}, d_{j}^{k}\right)$ drawn from the data posterior under the premise that data of different planets are independent. In order to obtain the data posterior samples $\left(d_{i}^{k}, d_{j}^{k}\right)^{\text {dep }}$ that incorporate the dependencies of data from different planets, we reject some of the original samples $\left(d_{i}^{k}, d_{j}^{k}\right)$. Those samples for which the ratio between dependent data prior and independent data prior is large (close to 1 or larger) are likely accepted as samples from $\left(d_{i}^{k}, d_{j}^{k}\right)^{\mathrm{dep}}$, while they are rejected if the ratio is closer to 0 . This procedure is done for all combinations of planets $i$ and $j$.

Alternatively, it is in principle possible to do an inference analysis by using all data and their correlations and interdependencies between planets. However, our scheme elegantly separates the information of correlated mass and radius of individual planets from information about interdependent data of different planets. This allows us to better control each analysis step and to be more flexible with testing different data scenarios without the need to run a full inference analysis for the entire system. Furthermore, our scheme can be generally applied to other inference problems.

\section{Results}

\subsection{Bulk Refractory Element Constraints}

In Figure 4, we show the consequences of different bulk constraints on mass and radius of TRAPPIST-1e. The prior data of measured mass and radius are highlighted in blue. The use of the suggested stellar constraint of Unterborn et al. (2017) (scenario $\mathrm{U}$ ) is not able to explain well the relatively high bulk density of TRAPPIST-1e. This is evident from the difference between the prior data distribution (blue) and the posterior distribution of scenario $U$ (green).

In a second scenario $\mathrm{N}$, we do not impose any abundance constraints. Instead, we analyze the possible range of bulk abundances of TRAPPIST-1e, while only using the data of mass, radius, and stellar irradiation. The predicted

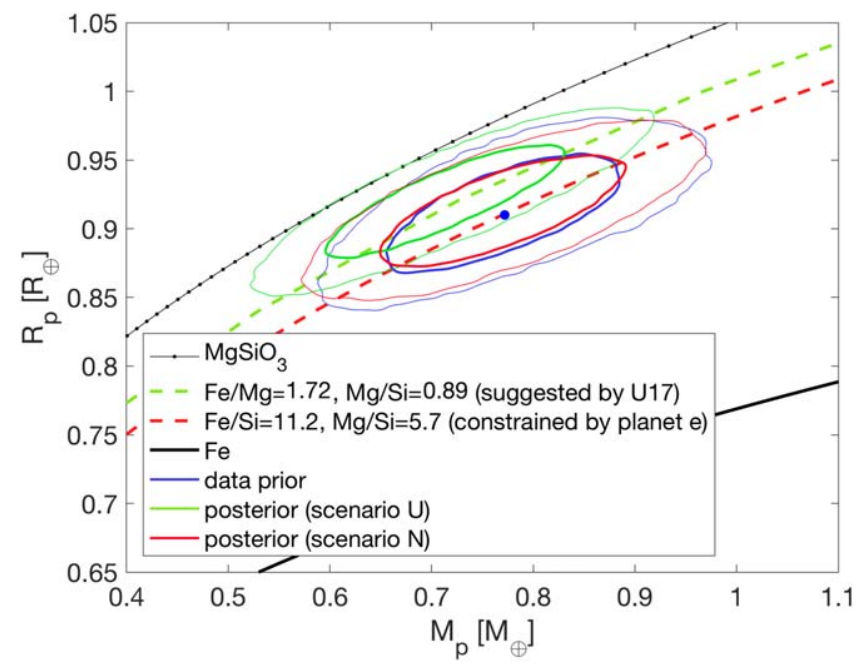

Figure 4. Comparison of data priors and data posteriors for TRAPPIST-1e for planetary mass and radius. The prior distribution (Grimm et al. 2018) is shown in blue, which overlaps well with the posterior distribution of scenario $\mathrm{N}$, but not only in parts with the posterior distribution of scenario U. In comparison, different mass-radius curves are plotted for pure iron (black solid) line, pure $\mathrm{MgSiO}_{3}$ (black solid line with circles), interiors of iron-free mantles that agree with the median constraint of Unterborn et al. (2017; green dashed line), and interiors of median structure and composition as inferred for planet $e$ and scenario N (red dashed line). Stated ratios are mass ratios.

bulk mass ratios are high, with large $1 \sigma$ uncertainties: $\mathrm{Fe} / \mathrm{Si}_{\mathrm{Tle}}=11.2 \pm 5.7$ and $\mathrm{Mg} / \mathrm{Si}_{\mathrm{Tle}}=5.7 \pm 3.7$. The data of only mass and radius (N) allow high relative bulk iron abundances, since iron incorporated in silicates and oxides in the mantle has a limited influence on bulk density. These abundance ranges $\left(\mathrm{Fe} / \mathrm{Si}_{\mathrm{Tle}}\right.$ and $\left.\mathrm{Mg} / \mathrm{Si}_{\mathrm{Tl}}\right)$ are subsequently used as input constraints to analyze the other planets in scenario A.

The ratios $\mathrm{Fe} / \mathrm{Si}_{\mathrm{Tle}}$ and $\mathrm{Mg} / \mathrm{Si}_{\mathrm{Tle}}$ are much higher than those estimated for Earth and other solar terrestrial planets. However, the large uncertainties on the ratios also allow for Earth-like rocky compositions. The fact that these uncertainties estimated from mass and radius alone are high illustrates the large degeneracy of rocky interiors, i.e., very different combinations of mantle compositions and core sizes can explain a given planetary mass and radius. The suggested stellar abundance proxy of Unterborn et al. (2017), with a relatively small uncertainty of $30 \%$, is partly incompatible with mass and radius of TRAPPIST-1e. In the discussion (Section 5), we provide two interpretations for the interior of planet e.

\subsection{Interior Predictions}

Our calculated interior predictions account for the variability in layer thicknesses (of core, mantle, ice/ocean, and gas), layer compositions (of mantle and gas), and thermal states. In the following, we present $1 \mathrm{D}$ and $2 \mathrm{D}$ marginalized distributions of estimated posteriors. Note that the $1 \sigma$ uncertainty regions differ between a distribution that is marginalized over one or two dimensions. The uncertainty regions are generally smaller for 1D marginalized distributions. Stated uncertainty regions refer to 1D marginalized distributions.

In Figure 5 we show the predicted ranges of radius fractions for gas and water layers for the scenario $U$, in which we use the stellar abundance proxy as suggested by Unterborn et al. (2017). Possible radius fractions of gas envelopes range 

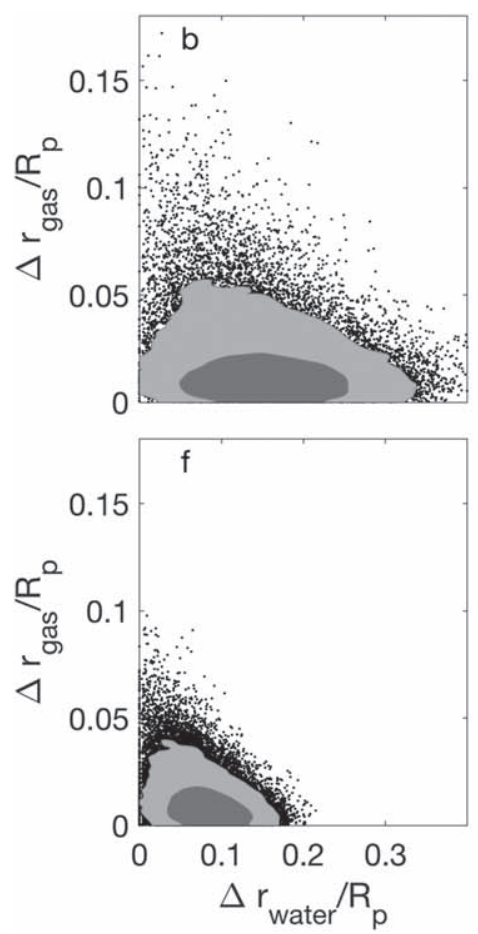
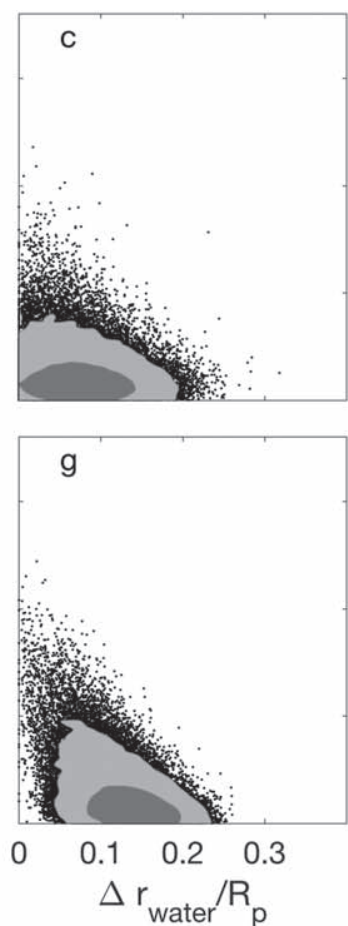
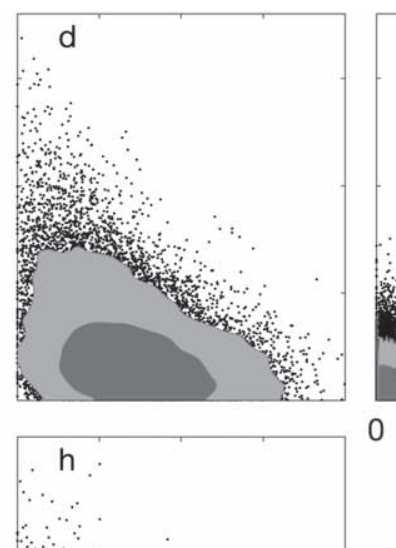

$$
\Delta r_{\text {water }}^{0.1} / R_{p} 0.3
$$

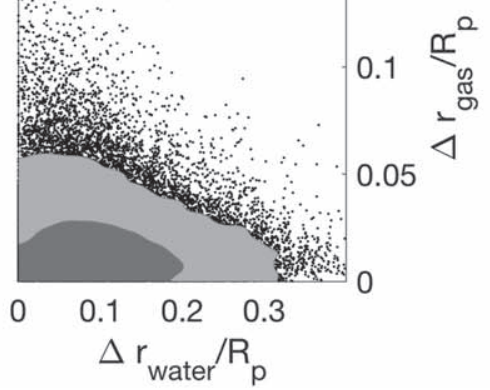$$
0
$$

Figure 5. 2D marginalized posterior distribution of gas and water radius fraction for scenario $\mathrm{U}$ and all seven planets (b-h). The difference in radius attributed to the gas or water layers is shown. Dark-gray and light-gray regions refer to $1 \sigma$ and $2 \sigma$ regions, respectively.
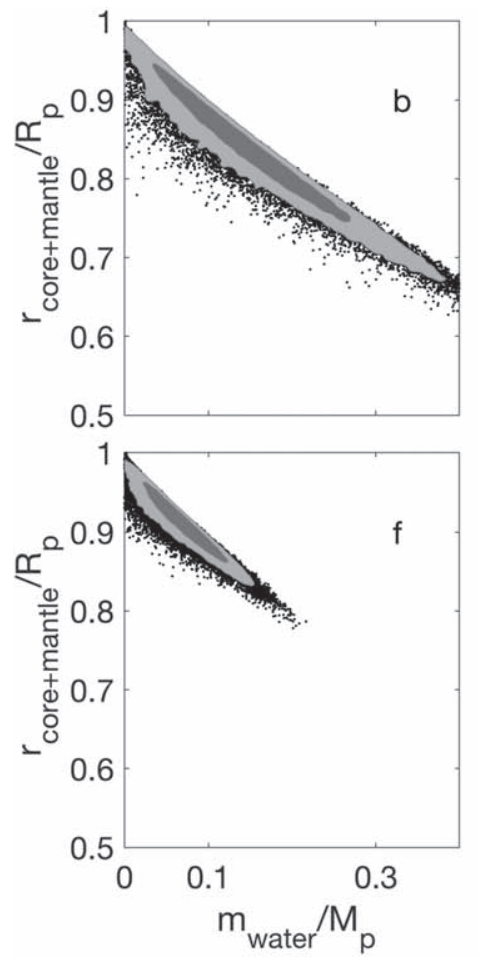
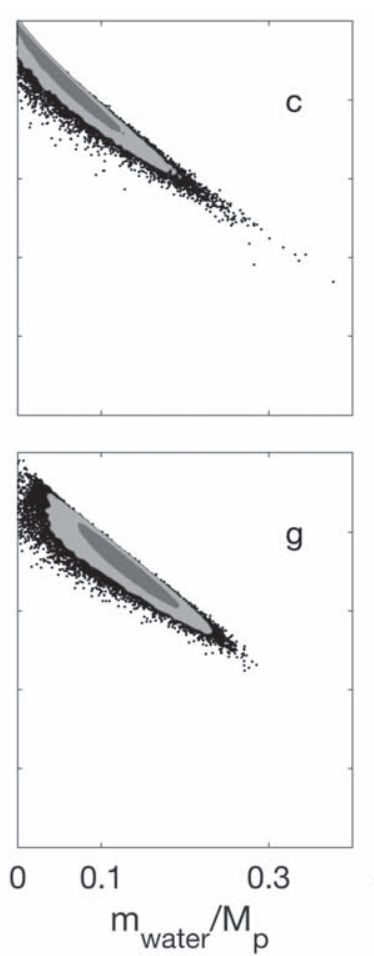
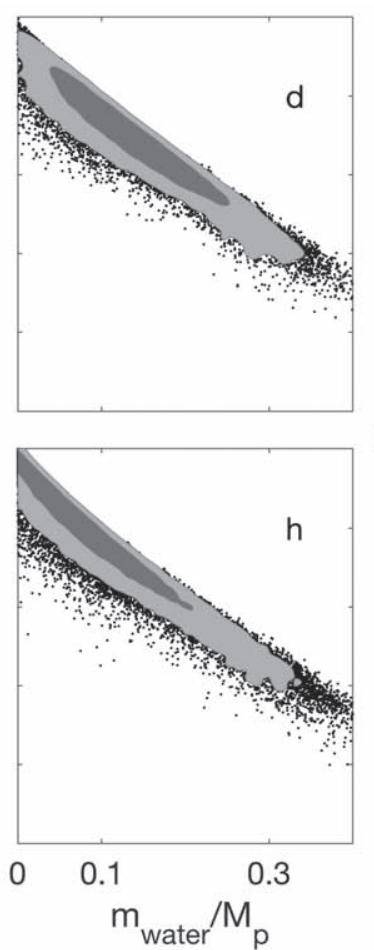

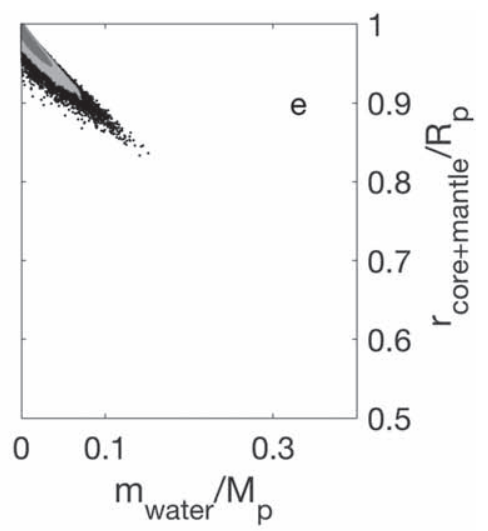

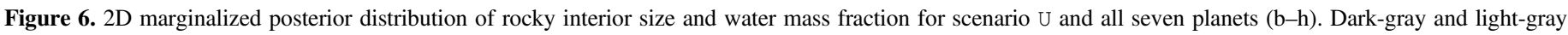
regions refer to $1 \sigma$ and $2 \sigma$ regions, respectively.

between $0 \%$ and $5 \%$, while water layers can contribute up to $30 \%$ to the total radius. The uncertainty ranges on possible amounts of water are large. This is because of the degeneracy with gas envelope thicknesses (Figure 5), but mainly the size of the rocky interiors (Figure 6) is large. There is a strong correlation between the possible amount of water and the size of the rocky interior, as expected.

In the following, we discuss how much the predicted water mass fractions depend on model assumptions and considered data. 


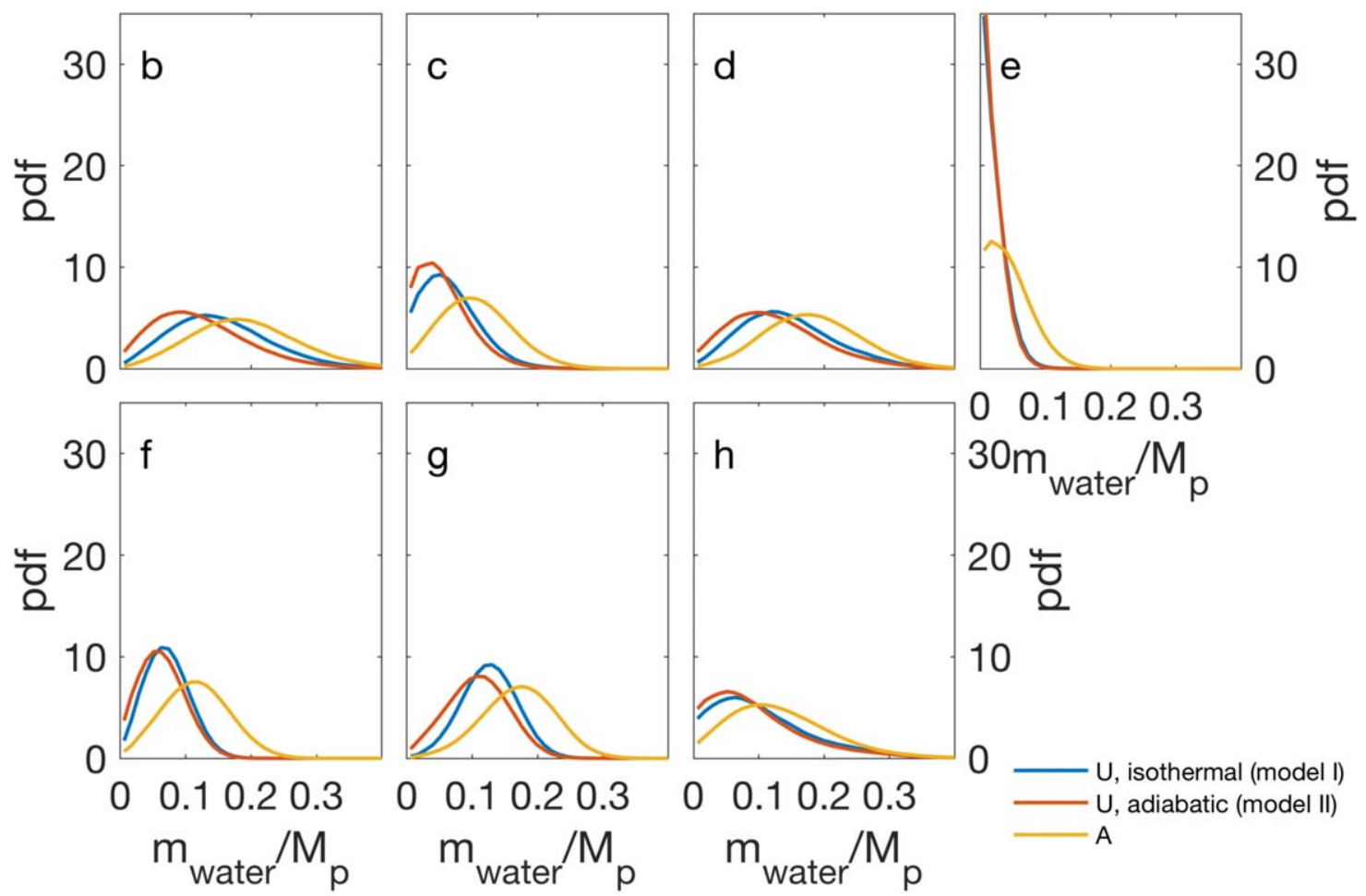

Figure 7. 1D marginalized posterior distribution of water mass fractions for all seven planets $(\mathrm{b}-\mathrm{h})$. The shown data scenarios use the stellar abundance proxy (U) or the abundance proxy based on TRAPPIST-1e (A). For the gas layer, model I (blue and yellow curve) assumes an isothermal temperature profile, while model II (red curve) assumes a convective adiabat in the gas layer.

Table 4

Interior Parameter Estimates with $1 \sigma$ Uncertainties of the 1D Marginalized Posterior Distributions

\begin{tabular}{|c|c|c|c|c|c|c|c|c|c|}
\hline \multirow{2}{*}{$\begin{array}{l}\text { Planet: } \\
\text { Scenario: }\end{array}$} & \multicolumn{3}{|c|}{ TRAPPIST-1b } & \multicolumn{3}{|c|}{ TRAPPIST-1c } & \multicolumn{3}{|c|}{ TRAPPIST-1d } \\
\hline & $\mathrm{U}$ & A & UCM & $\mathrm{U}$ & A & UCM & $\mathrm{U}$ & A & UCM \\
\hline$r_{\text {core }} / r_{\text {core }+ \text { mantle }}$ & $0.39_{-0.11}^{+0.09}$ & $0.50_{-0.14}^{+0.12}$ & $0.39_{-0.11}^{+0.09}$ & $0.40_{-0.11}^{+0.08}$ & $0.52_{-0.13}^{+0.11}$ & $0.40_{-0.11}^{+0.08}$ & $0.39_{-0.11}^{+0.09}$ & $0.51_{-0.14}^{+0.12}$ & $0.39_{-0.12}^{+0.08}$ \\
\hline$r_{\text {core }+ \text { mantle }} / R_{\mathrm{p}}$ & $0.84_{-0.06}^{+0.06}$ & $0.79_{-0.07}^{+0.07}$ & $0.82_{-0.07}^{+0.08}$ & $0.92_{-0.05}^{+0.04}$ & $0.86_{-0.06}^{+0.06}$ & $0.91_{-0.05}^{+0.04}$ & $0.84_{-0.06}^{+0.05}$ & $0.79_{-0.06}^{+0.06}$ & $0.84_{-0.06}^{+0.06}$ \\
\hline$m_{\text {water }} / M_{\mathrm{p}}$ & $0.15_{-0.07}^{+0.08}$ & $0.19_{-0.08}^{+0.09}$ & $0.16_{-0.09}^{+0.10}$ & $0.06_{-0.04}^{+0.05}$ & $0.10_{-0.05}^{+0.06}$ & $0.06_{-0.04}^{+0.05}$ & $0.14_{-0.06}^{+0.08}$ & $0.18_{-0.07}^{+0.07}$ & $0.14_{-0.07}^{+0.09}$ \\
\hline$r_{\text {water }} / R_{\mathrm{p}}$ & $0.15_{-0.06}^{+0.07}$ & $0.19_{-0.07}^{+0.07}$ & $0.16_{-0.08}^{+0.07}$ & $0.07_{-0.04}^{+0.05}$ & $0.12_{-0.06}^{+0.06}$ & $0.08_{-0.04}^{+0.05}$ & $0.14_{-0.06}^{+0.06}$ & $0.18_{-0.06}^{+0.06}$ & $0.13_{-0.06}^{+0.07}$ \\
\hline$r_{\mathrm{gas}} / R p$ & $0.01_{-0.01}^{+0.01}$ & $0.01_{-0.01}^{+0.01}$ & $0.01_{-0.01}^{+0.01}$ & $0.01_{-0.01}^{+0.01}$ & $0.01_{-0.01}^{+0.01}$ & $0.01_{-0.01}^{+0.01}$ & $0.02_{-0.01}^{+0.02}$ & $0.02_{-0.01}^{+0.02}$ & $0.02_{-0.01}^{+0.02}$ \\
\hline Planet: & \multicolumn{3}{|c|}{ TRAPPIST-1e } & \multicolumn{3}{|c|}{ TRAPPIST-1f } & \multicolumn{3}{|c|}{ TRAPPIST-1g } \\
\hline Scenario: & $\mathrm{U}$ & A & UCM & $\mathrm{U}$ & A & UCM & $\mathrm{U}$ & A & UCM \\
\hline$r_{\text {core }} / r_{\text {core }+ \text { mantle }}$ & $0.45_{-0.10}^{+0.06}$ & $0.58_{-0.11}^{+0.09}$ & $0.45_{-0.10}^{+0.07}$ & $0.39_{-0.11}^{+0.08}$ & $0.52_{-0.13}^{+0.12}$ & $0.39_{-0.11}^{+0.08}$ & $0.39_{-0.11}^{+0.08}$ & $0.50_{-0.14}^{+0.12}$ & $0.39_{-0.11}^{+0.09}$ \\
\hline$r_{\text {core }+ \text { mantle }} / R_{\mathrm{p}}$ & $0.97_{-0.02}^{+0.02}$ & $0.93_{-0.05}^{+0.04}$ & $0.97_{-0.02}^{+0.02}$ & $0.91_{-0.03}^{+0.03}$ & $0.86_{-0.05}^{+0.05}$ & $0.91_{-0.03}^{+0.03}$ & $0.86_{-0.03}^{+0.03}$ & $0.81_{-0.05}^{+0.05}$ & $0.86_{-0.03}^{+0.03}$ \\
\hline$m_{\text {water }} / M_{\mathrm{p}}$ & $0.02_{-0.01}^{+0.02}$ & $0.04_{-0.03}^{+0.04}$ & $0.02_{-0.01}^{+0.02}$ & $0.07_{-0.03}^{+0.03}$ & $0.11_{-0.05}^{+0.05}$ & $0.07_{-0.03}^{+0.03}$ & $0.13_{-0.04}^{+0.04}$ & $0.17_{-0.06}^{+0.05}$ & $0.13_{-0.04}^{+0.04}$ \\
\hline$r_{\text {water }} / R_{\mathrm{p}}$ & $0.02_{-0.01}^{+0.02}$ & $0.06_{-0.04}^{+0.05}$ & $0.02_{-0.01}^{+0.02}$ & $0.08_{-0.03}^{+0.03}$ & $0.13_{-0.05}^{+0.05}$ & $0.08_{-0.03}^{+0.03}$ & $0.13_{-0.04}^{+0.03}$ & $0.18_{-0.06}^{+0.05}$ & $0.13_{-0.04}^{+0.04}$ \\
\hline$r_{\mathrm{gas}} / R p$ & $0.01_{-0.00}^{+0.01}$ & $0.01_{-0.01}^{+0.01}$ & $0.01_{-0.00}^{+0.01}$ & $0.01_{-0.01}^{+0.01}$ & $0.01_{-0.01}^{+0.01}$ & $0.01_{-0.01}^{+0.01}$ & $0.01_{-0.01}^{+0.01}$ & $0.01_{-0.01}^{+0.01}$ & $0.01_{-0.01}^{+0.01}$ \\
\hline Planet: & \multicolumn{3}{|c|}{ TRAPPIST-1h } & & & & & & \\
\hline Scenario: & $\mathrm{U}$ & A & UCM & & & & & & \\
\hline$r_{\text {core }} / r_{\text {core }+ \text { mantle }}$ & $0.40_{-0.11}^{+0.08}$ & $0.52_{-0.14}^{+0.11}$ & $0.40_{-0.12}^{+0.08}$ & & & & & & \\
\hline$r_{\text {core }+ \text { mantle }} / R_{\mathrm{p}}$ & $0.89_{-0.07}^{+0.06}$ & $0.84_{-0.07}^{+0.07}$ & $0.87_{-0.07}^{+0.06}$ & & & & & & \\
\hline$m_{\text {water }} / M_{\mathrm{p}}$ & $0.09_{-0.06}^{+0.09}$ & $0.12_{-0.07}^{+0.08}$ & $0.11_{-0.06}^{+0.09}$ & & & & & & \\
\hline$r_{\text {water }} / R_{\mathrm{p}}$ & $0.10_{-0.06}^{+0.08}$ & $0.14_{-0.07}^{+0.08}$ & $0.11_{-0.06}^{+0.08}$ & & & & & & \\
\hline$r_{\mathrm{gas}} / R p$ & $0.01_{-0.01}^{+0.02}$ & $0.01_{-0.01}^{+0.02}$ & $0.01_{-0.01}^{+0.02}$ & & & & & & \\
\hline
\end{tabular}

\subsubsection{Influence of the Choice of Abundance Proxy}

Figure 7 illustrates how the choice of abundance constraints influences the predicted amount of water. If the stellar proxy (U) is used, rocky interiors are less dense compared to the proxy that is based on TRAPPIST-1e (A). Consequently, the predicted amount of water is smaller in scenario $U$, since less water can be added on top of low-density rocky interiors while still fitting mass and radius. There are differences in the median 


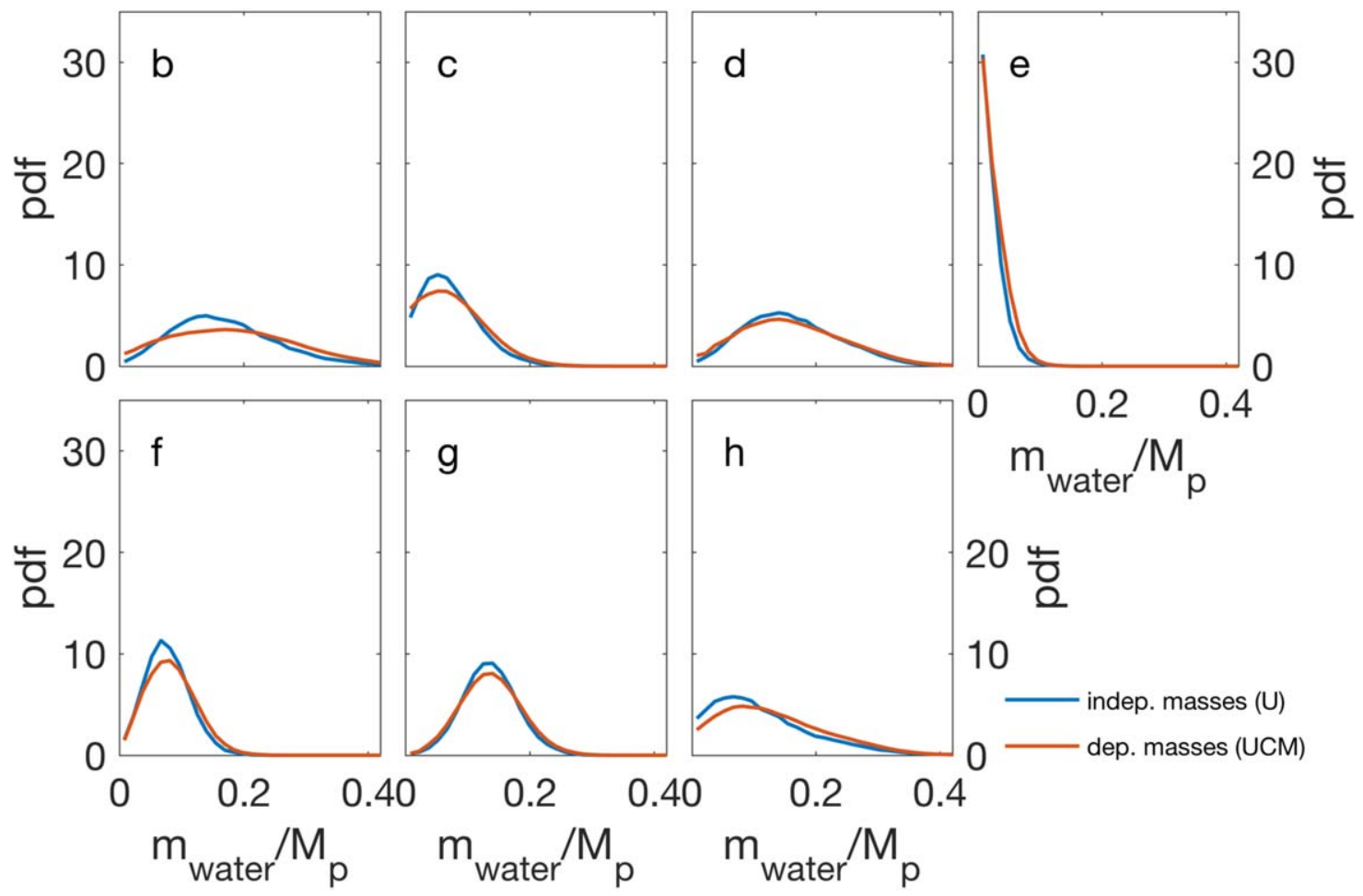

Figure 8. 1D marginalized posterior distribution of water mass fractions for all seven planets $(b-h)$. U ignores interdependencies of planetary data, while UCM accounts for interdependent masses. The change between U and UCM depends on the abundance proxy used. Shown scenarios use the stellar proxy.

predicted water mass fraction that range from $25 \%$ up to $50 \%$ between $U$ and $A$. For all but planet e, the differences are largely around $30 \%$. For planet e, it is the discrepancy between mass and radius versus stellar proxy that leads to such large changes in $m_{\text {water }} / M_{\mathrm{p}}$ between the scenarios $\mathrm{U}$ and A. Table 4 summarizes the estimated interior parameters and their $1 \sigma$ uncertainties.

\subsubsection{Accounting for the Interdependency of Planetary Masses}

Inferred planetary masses by Grimm et al. (2018) are significantly correlated between different planets (Figure 2). We account for this interdependency of planetary masses by resampling the posterior interior samples of scenario $U$ with our new resampling scheme (Section 3.4.3) that yields the posterior distribution UCM. The difference between the posterior distribution $U$ and UCM demonstrates the information contained in the interdependency of planetary masses. Figure 8 shows that the differences are largest for planets $\mathrm{b}$ and $\mathrm{h}$, with up to $20 \%$ difference in both the median water mass fraction and the corresponding uncertainty (Table 4).

The information contained in the interdependency of planetary data depends on the specific correlation between the planets. In Figure 9 we show how the distribution of water mass fraction for planet $b$ changes while adding step by step the information on the correlation between the planetary masses. For planet $b$, the information gained by accounting for interdependency of masses is mainly kept in the interdependency with planets e and h. In general, how much information can be gained depends on the actual level of interdependency of planet pairs. While accounting for interdependent data, water mass fractions are shifting toward lower values, and uncertainties increase.

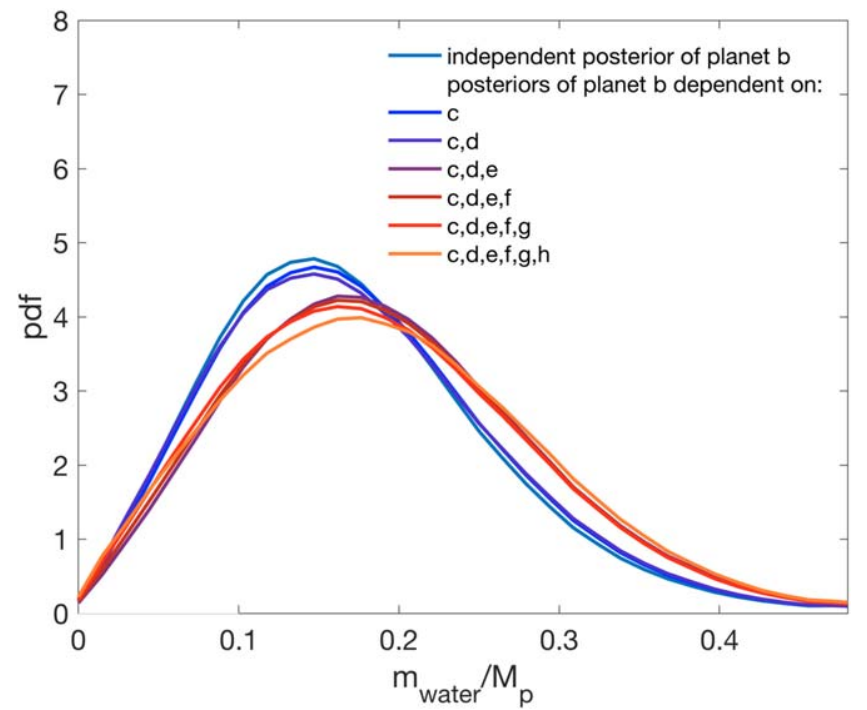

Figure 9. 1D marginalized posterior distribution of water mass fractions for planet $\mathrm{b}$. The independent posterior of scenario $U$ (light blue line) yields the highest water mass estimates. While subsequently taking into account the interdependency of planetary masses between planet $b$ and the other planets, the curves are colored with more reddish colors and move to lower water mass fractions. The lowest water mass fractions represent scenario UCM.

Why do uncertainties on estimated water mass fractions increase? The reason is illustrated in Figure 10, showing the independent posteriors (U) and the prior ratio $\left(\rho_{d_{i j}}\left(\boldsymbol{d}_{i}, \boldsymbol{d}_{j}\right) / Q \rho_{d_{i}}\left(\boldsymbol{d}_{i}\right) \rho_{d_{j}}\left(\boldsymbol{d}_{j}\right)\right.$ from Equation (13)), which is used to resample from the independent posteriors in order to obtain dependent posteriors. The ratio is highest at the two tails of the 

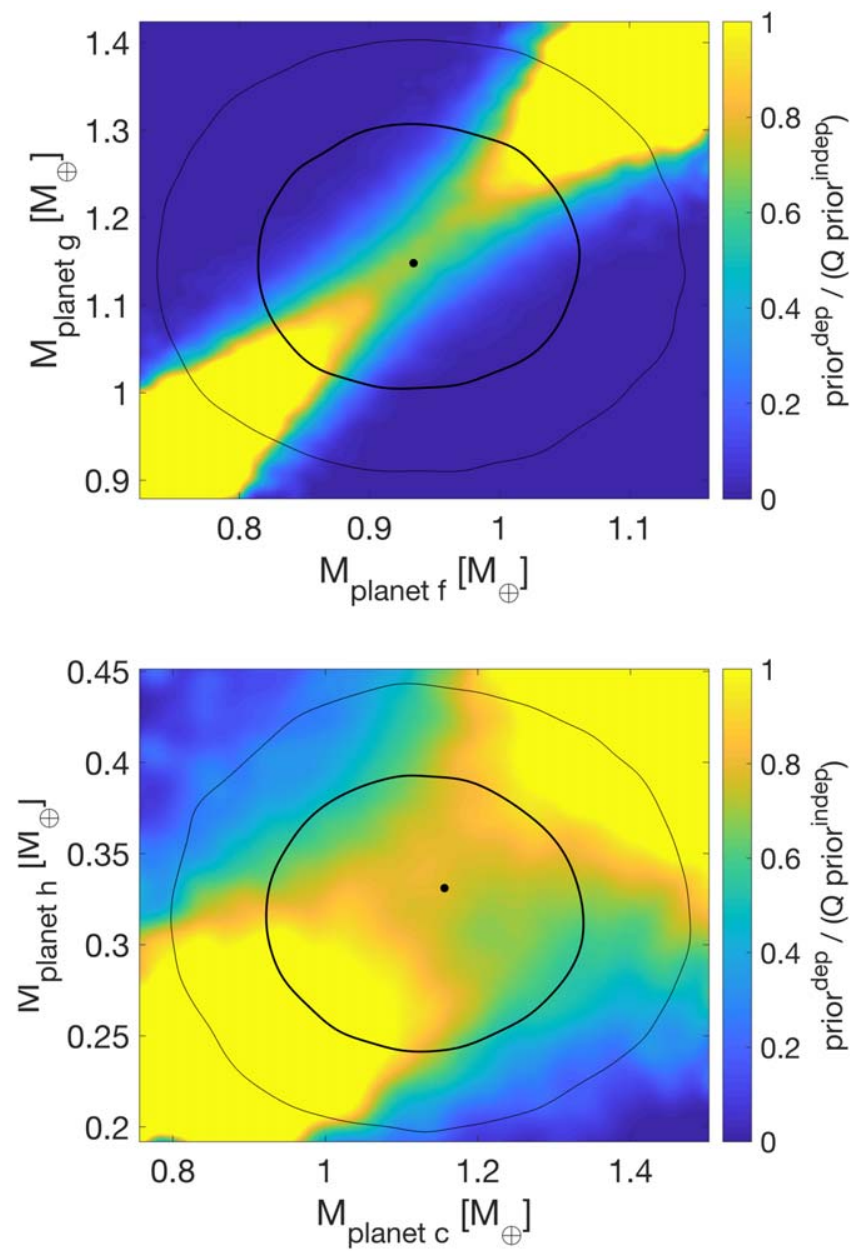

Figure 10. Planetary mass distributions for planets $\mathrm{f}$ and $\mathrm{g}$ (top panel) and planets $\mathrm{c}$ and $\mathrm{h}$ (bottom panel). The $1 \sigma$ and $2 \sigma$ contours in white are the independent posteriors of scenario $U$. These posteriors are resampled according to the ratio prior $^{\text {dep }} /\left(Q\right.$ prior $\left.^{\text {indep }}\right)$ (in color) as described in Section 3.4.3 in order to obtain the dependent posteriors.

2D distributions, where planetary masses are both small and large. These tails of the independent mass distribution are preferentially resampled. For a given radius, high masses are characterized by low water contents and vice versa. In consequence, the distribution of possible water mass fractions broadens.

Why are higher water mass fractions preferentially resampled? For some of the planets, the highest possible masses cannot be well described by the stellar proxy (U). This is the case for planet e (as discussed earlier for Figure 4) but also planets c and h (Figure 10, bottom panel). It is not the case, for example, for planets $\mathrm{f}$ and $\mathrm{g}$ (Figure 10, top panel). The consequence is clear from the posteriors $(\mathrm{U})$ that are shifted to smaller planetary masses compared to the prior data (for e, $\mathrm{h}$, and c). During the resampling of the posterior $\mathrm{U}$, only those samples survive for which the prior ratio is large. This is the case for lower masses (Figure 10, bottom panel) that are characterized by higher water contents (for a given radius).

The difference between $U$ and UCM is due to not only the partial incompatibility of planetary data (mass and radius versus the stellar proxy) of planets $\mathrm{e}, \mathrm{h}$, and $\mathrm{c}$ but also the fact that the 2D mass distributions (Figure 2) are not perfect ellipses.

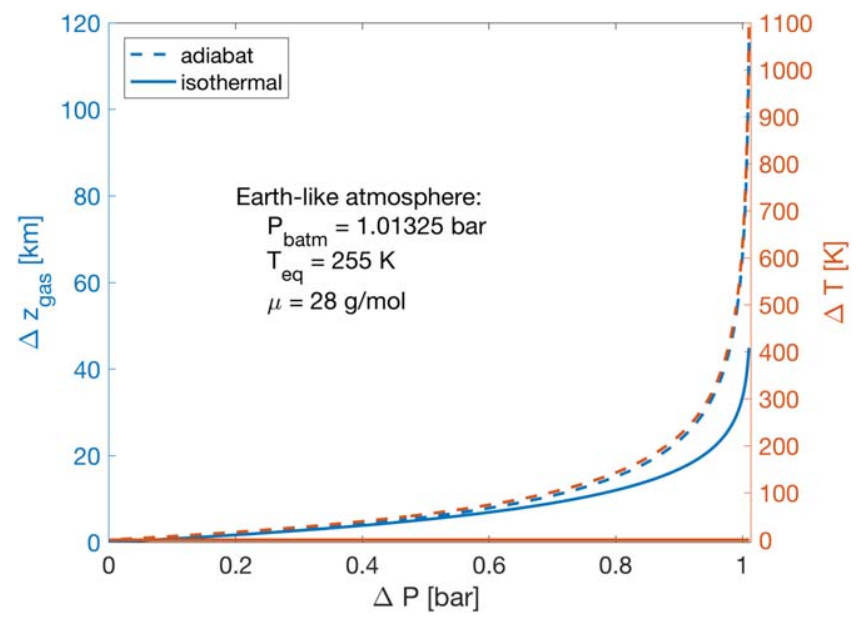

Figure 11. Comparison of atmospheric models I (isothermal temperature profile; solid lines) and II (adiabatic; dashed lines) for an Earth-like atmosphere. $\Delta z_{\text {gas }}$ is the depth into the gas envelope reaching different pressures $\Delta P$ compared to $20 \mathrm{mbar}$ at $z_{\mathrm{gas}}=0 \mathrm{~km}$. Depending on the model (I or II), the increase in temperature $\Delta T$ is zero or higher than Earth's atmospheric temperatures. The models enclose Earth's average atmosphere profile and represent end-member cases.

\subsubsection{Influence of Atmospheric Model}

Figure 7 shows that the differences in water mass fractions are small when we change from an isothermal to a fully adiabatic temperature model for the gas layer. In the isothermal model I, we have introduced a parameter $\alpha$ that accounts for cooling and heating of an atmosphere. However, temperatures are largely limited to $400 \mathrm{~K}$, and thus, especially for the innermost planets, this model I is not able to produce temperatures as high as suggested by Grimm et al. (2018) (surface temperatures of $750-1500 \mathrm{~K}$ for planet b). Model II overcomes this limitation but overpredicts the temperatures in the gas layer by assuming a fully convective gas layer. In consequence, surface temperatures in model II can be very high and can range, for example, on planet b from 500 to $10,000 \mathrm{~K}$ for surface pressures of $1-10^{4}$ bars. Thus, while temperatures in model I are underestimated, they are overestimated in model II. This is illustrated in Figure 11, showing pressure-temperature profiles for an Earth-like atmosphere. Global climate models that solve for radiative transfer as presented in Turbet et al. (2018) yield much more realistic surface temperatures. However, we find that there is only limited influence due to the choice of gas layer model on the estimated mass of the underlying water layer. Median estimates generally vary by $10 \%-15 \%$, expect for planets $\mathrm{b}$ and $\mathrm{c}$ with $25 \%$. The differences are relatively small compared to other influences (Sections 4.2.1 and 5.1).

Estimated water mass fractions are smaller for model II compared to model I. This is because the lower gas envelope temperatures in model I result in smaller scale heights and thin gas envelopes, and thus larger amounts are required to fit planet mass and radius. The solutions given the two models I and II represent end-member solutions.

\subsubsection{Relevance of Data Uncertainty}

Figure 12 illustrates how much our ability to constrain water mass fraction would improve by more precise estimates of mass and radius. An improved estimate on planet mass (70\% of the nominal uncertainty) only has generally marginal influence 

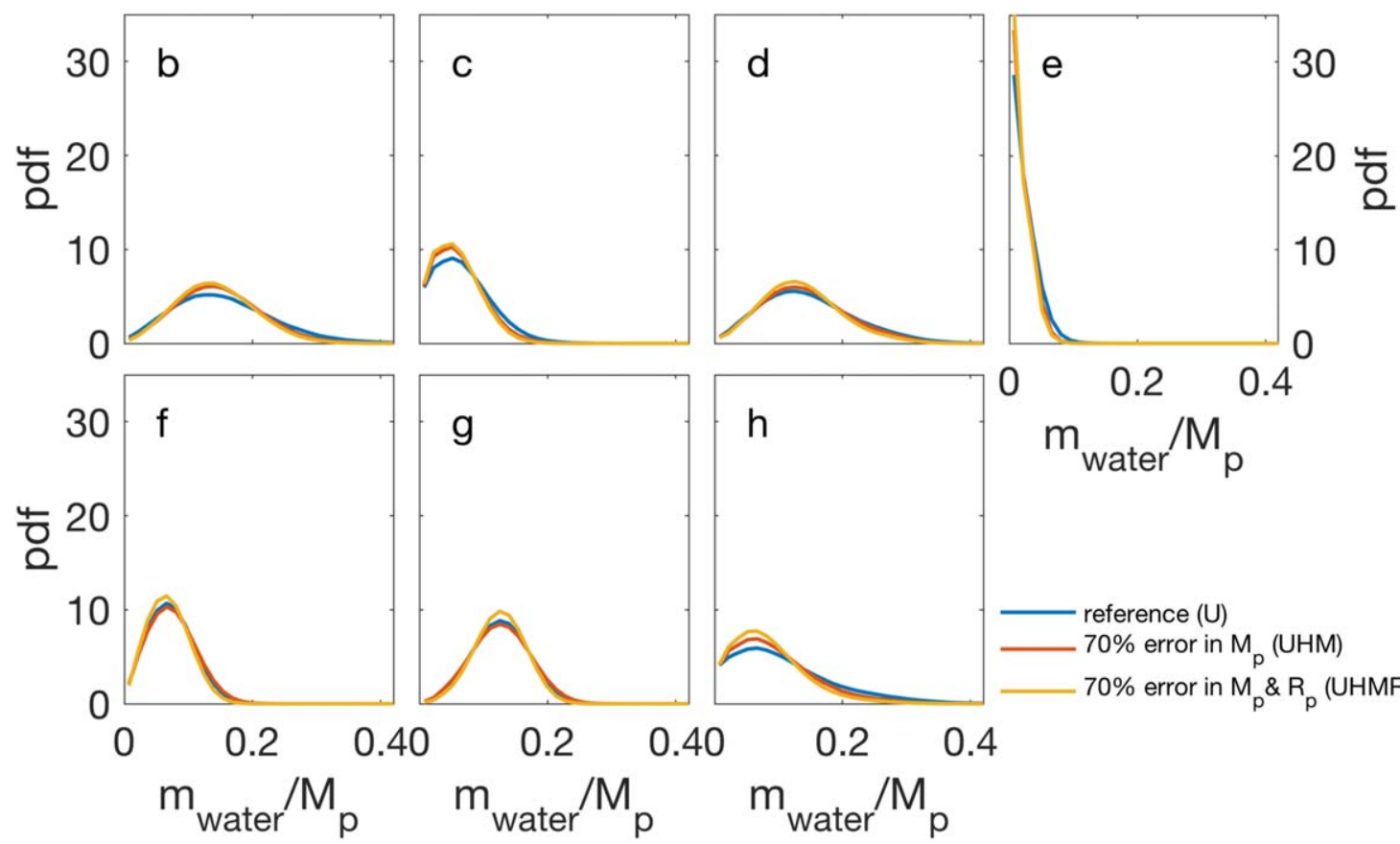

Figure 12. 1D marginalized posterior distribution of water mass fractions for all seven planets $(b-h)$. For $U$ the nominal data uncertainties are used, while for UHM and UHMR 70\% of the uncertainties on planet mass and on mass and radius are used, respectively. All shown data scenarios use the stellar proxy.

on the median estimate of $m_{\text {water }} / M_{\mathrm{p}}$, but it reduces the $1 \sigma$ confidence interval by $5 \%-20 \%$. Improved estimates on both planet mass and radius ( $70 \%$ of the nominal uncertainty) reduce the $1 \sigma$ confidence interval by $10 \%-35 \%$. The largest improvements are seen for planets $\mathrm{e}$ and $\mathrm{h}$, while the smallest improvements are seen for planets $f$ and $g$. The information gained by improved data uncertainty is small compared to the different choices of abundance proxies (Figure 7), as well as the information kept in the interdependency of planetary data (Figure 8).

\section{Discussion}

Although masses, radii, and temperature conditions of the TRAPPIST-1 planets generally remind us of terrestrial solar system planets, the possible interiors of the TRAPPIST-1 planets can contain significant water budgets of up to $20 \%$ or even $30 \%$, unlike the terrestrial planets $(\leqslant 0.02 \%$ surface water). This is because of the roughly $20 \%$ lower bulk densities, their associated uncertainties, and the large degeneracy due to variability in structure and composition of the rocky interior, as well as in gas layer characteristics.

\subsection{Systematic Data Biases}

Recent announcements (Demory 2018) have indicated that previously unavailable observations for the TTV analysis of Grimm et al. (2018) provide a higher accuracy with changes in planetary masses within $\pm 24 \%$. In Figure 13 , we show that consequences for the estimated water mass fractions are large. Planet e would not be the odd case of a super-Mercury. All planets could be consistent with an increasing water mass fraction with orbital period (within $2 \%-12 \%$ with respect to $1 \sigma$ errors) or a uniform water mass fraction of $7 \%$ (within $1 \sigma$ errors). Such large amounts of water would exclude Earth-like habitability even for the temperate planets since their rocky interiors are separated by icy layers from the water oceans in that case. In contrast, the published data estimates (Grimm et al. 2018) suggest no clear trend of water mass fraction with orbital distance as discussed below (Figure 13).

Specifically for the TRAPPIST-1 planets, the trend of changing water mass fractions with systematic shifts in planetary masses can be described as follows. A shift of $+25 \%$ in planetary mass can reduce the water mass fraction by $65 \%$ (i.e., the reference water mass fraction multiplied by a factor of 0.35 ). For shifts of $+20 \%,+10 \%,+5 \%$, and $-15 \%$ the factor is roughly $0.4,0.55,0.75$, and 2.6 , respectively.

In general, biases from the TTV analysis are extremely difficult to quantify because they stem from limited observations. Biases from TTV can be caused by unresolved degeneracies between eccentricity, mass, and arguments of perihelion of the different planets. Limited observation time can cause the TTV analysis to prefer solutions that depart from the true solution, or the parameter search is trapped in a local minimum of the sampling parameter space. Also strong tidal effects, nearby perturbing stars, or even yet-undetected additional planets can introduce a TTV signal bias. Further TTV analysis on a larger number of transit observations is needed to increase the accuracy of planetary masses.

\subsection{Rocky Interiors and TRAPPIST-1e}

The degeneracies within structure and composition of rocky interiors stem from the fact that we allow for variable silicate mantle compositions and core sizes. Independent constraints on element abundances as taken from stellar proxies are highly valuable for refined rocky interior estimates (Dorn et al. 2015). Here, however, no direct estimates from the faint host star are available. Instead, we have adopted two different abundance proxies (see Table 3): a stellar proxy (scenario $\mathrm{U}$ ) as derived from F-G-K stars of similar metallicities (Unterborn et al. 2017), and a proxy based on only mass and radius of the densest planet of the system, planet e (scenario A). 
Depending on the abundance proxies used for refractory elements ( $U$ or $A)$, the interior estimates change with significant influences on the predicted possible water budgets (Section 4.2.1). Any decision on whether scenario $U$ or $A$ is more likely depends on the interpretation of planet e.

If TRAPPIST-1e were a super-Mercury-type planet, it would not represent the rocky analog for all planets. In this case, scenario A would be best only to describe planet e, while all other planets would be best described by scenario UCM (in case the stellar proxy would be excluded for planet e). A superMercury-type interior could be explained by a high-energy giant impact (Benz et al. 1988; Stewart \& Leinhardt 2009; Marcus et al. 2010). This would also allow us to explain why inferred interiors for planet e are poorest in volatiles, although its neighboring planets are volatile-rich. A mantle-stripping impact would require large impact velocities or small impact angles (Stewart \& Leinhardt 2009), or an impactor larger than planet e, which does not exist in the system. It remains to be studied whether impacts are a reasonable scenario.

If TRAPPIST-1e is not a super-Mercury-type planet, there are two interpretations possible. First, TRAPPIST-1e can indeed be a rocky analog of all the planets regarding the relative ratios of rock-forming elements. In this case, all planets have compositions that are not Earth-like, implying that the iron was more abundant compared to solar compositions. In this case, all planetary interiors are best described by scenario A.

Second, let us assume that TRAPPIST-1e is indeed similar to an Earth interior and thus well represented by $U$, but its mass and radius estimates are affected by systematic biases (as discussed in Section 5.1). In this case, scenario U may well describe planet e, while dismissing high bulk density interiors. Thus, many mass-radius realizations are incompatible for planet e in scenario U. Given that all planetary masses (radii) are correlated to the mass (radius) of planet e, there are also many planetary masses (radii) of the other planets that are incompatible with U. In consequence, accounting for the interdependencies of planetary data improves interior estimates. If this case is true, all interiors are well described by scenario UCM.

\subsection{Water Budgets}

The layers of water can generally be in liquid, solid, and even supercritical state. Water vapor is excluded, as we impose that any water layer requires a gaseous layer on top that imposes a pressure at least as high as the vapor pressure of water. For simplification, we use pure water and neglect other forms of ices (e.g., $\mathrm{CO}_{2}$ ). For all but planet e, most of the possible planetary interiors are characterized by ice layers separating water oceans from rocky interiors. This is due to the fact that either the large amounts of water allow pressures high enough to form high-pressure ices or temperatures are sufficiently cool (especially for the outer planets $\mathrm{f}, \mathrm{g}$, and $\mathrm{h}$ ). We note that the thermal states of the planets are unknown and significant tidal heating may allow for more extended thicknesses of liquid water. Planet e is the volatile-poor exception among the planets. Its possible tiny amounts of water and temperate conditions allow for liquid water on the surface. Also for planets $\mathrm{c}$ and $\mathrm{h}$, the uncertainty on mass and radius allows for negligibly thin volatile layers.

In Figure 14, we show the $2 \sigma$ uncertainty distribution for scenario $U$ and all planets in comparison with mean estimates for Earth and Venus, but also Titan and icy satellites, which probably formed outside the water ice line: Europa, Ganymede, Callisto, Triton, Pluto, and Charon. The water mass fractions of the shown solar system objects range from $0 \%$ to $30 \%$ (for a review, see Nimmo \& Pappalardo 2016) and are comparable to our inferred ranges of possible water mass fractions. Water mass fractions of solar system objects on the order of $1000 \mathrm{~km}$ or smaller can have higher water budgets. (Saturn's moon Tethys, with a radius of $1060 \mathrm{~km}$, is almost entirely composed of water, with a bulk density of $0.98 \mathrm{~g} \mathrm{~cm}^{-3}$.) Formation models that simulate the structure and evolution of disks (Alibert \& Benz 2017) predicted planets to be more volatilerich around low-mass stars, since the snow line is located closer to the stars compared to the solar system. Indeed, the TRAPPIST-1 system is significantly more volatile-rich than the terrestrial solar system planets.

Our results suggest an upper water mass fraction of not much larger than $30 \%$. The amount of water loss (Bourrier et al. 2017) or water delivery (Kral et al. 2018) during the longterm evolution of the planets after formation is small compared to our predicted uncertainties on water budgets. While water budgets can be altered by 1-100 Earth oceans, the predicted water budgets range from tens to thousands of Earth oceans, with uncertainties on the scale of hundreds to thousands of Earth oceans. Therefore, our inferred water budgets largely represent the water budgets originally accreted during formation.

In comparison to the expected water mass fraction of material outside the water snow line of 50\% (Lodders 2003), our maximum predicted water budgets are significantly smaller $(30 \%)$. This can be due to a mixed accretion of volatile-poor and volatile-rich planetesimals from within and beyond the ice line, respectively. Sufficient mixing of volatile-poor and volatile-rich planetesimals for all planets is also reasonable given that no clear trend of water budget with orbital distance is seen as shown in Figure 13. Here we plot the inferred water mass fractions against the equilibrium temperature at zero albedo, which relates to orbital distance. The error bars on water mass fraction represent 3th-16th-84th-97th percentiles of the cumulative distribution. Neither a clear trend of water mass fraction with orbital distance nor one with planet mass could be identified. Thus, our findings suggest that the accreted material stems from regions in the disk where mixing was efficient enough to blur any possible trends of increasing water mass fraction with orbital distance, unless migration reordered the planets. Below, we discuss the implications for the formation of the planets in more detail.

\subsection{Implications for Planet Formation}

The two main conclusions of our calculations are that the water content is very diverse among the planets, with no obvious correlation with either the mass or the period, and that the maximum amount of water is somewhat lower than the icy content of planetesimals usually assumed in the solar system.

The scatter in planetary water budgets is at odds with what we observe in the solar system, both in the planets and in Galilean satellites. In both cases, innermost objects tend to be dryer than outermost ones. While a uniform water budget in all planets cannot be excluded at the $2 \sigma$ level (see Figure 13), this scatter is puzzling. Different origins can be invoked for this scatter. First, planets likely migrated during their formation (Alibert \& Benz 2016), and therefore accreted planetesimals 

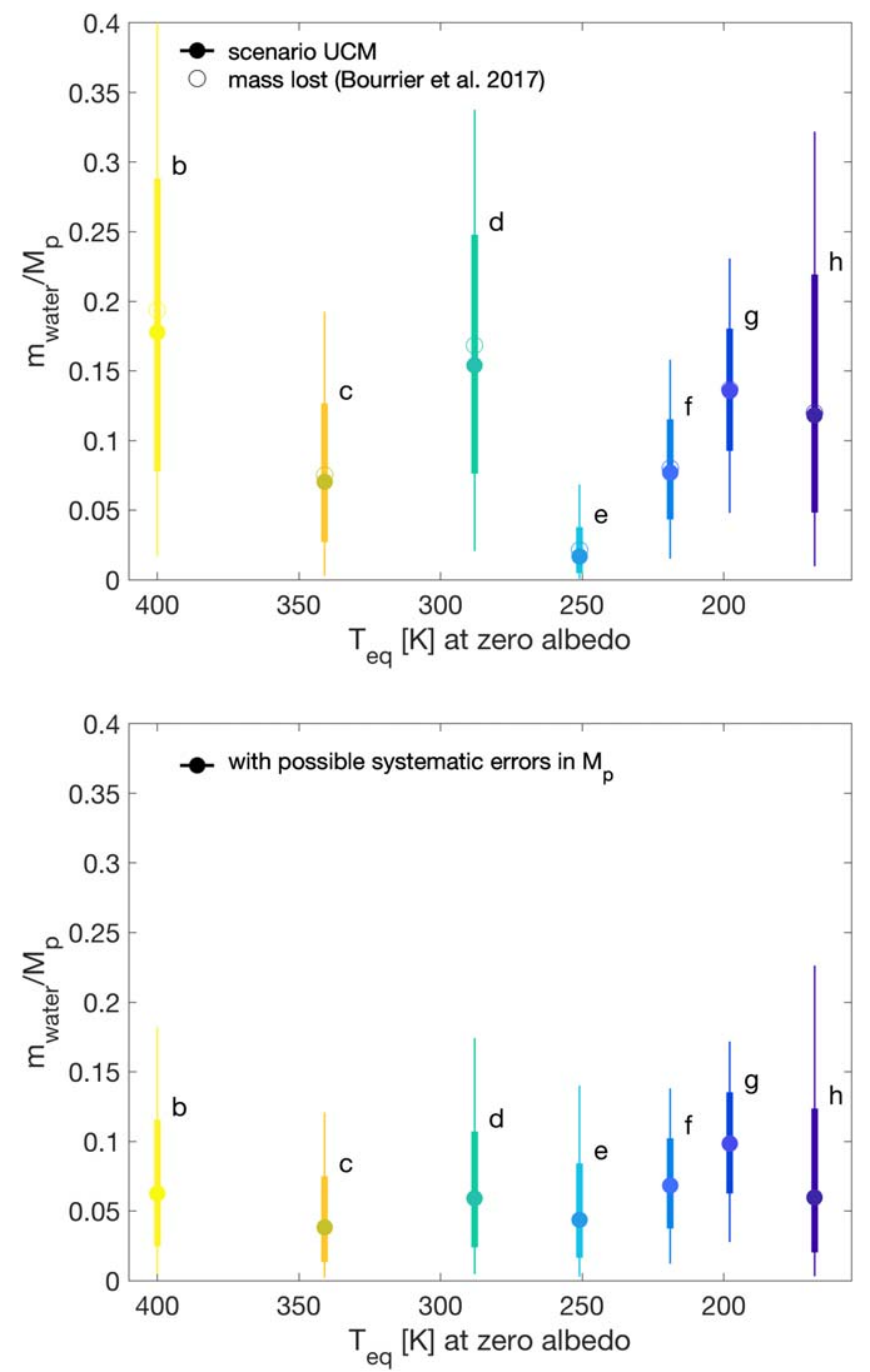

Figure 13. Marginalized water mass fractions as a function of equilibrium temperature $T_{\mathrm{eq}}$ (at zero albedo). The top panel shows scenario UCM. There is no obvious trend of increasing water mass fraction with larger orbital distance and thus cooler $T_{\text {eq }}$. For the water mass fractions, the 3th-16th-84th-97th percentiles are depicted by the thin and thick error bars. How much water mass fraction there could have been after formation (open circles) is calculated by adding the amount of lost water (Bourrier et al. 2017) to our inferred median estimates (filled circles). The bottom panel shows that possible systematic shifts in planet masses (from $-18 \%$ to $24 \%$ ) could in fact result in finding water mass fractions that are uniform or increasing with orbital distance.

from different parts in the disk, some dry (inside the ice line) and some wet (beyond the ice line). In addition, the ice line itself is likely to move during the formation of planets, and this can modify the water content of solids in the disk (pebbles, but also small planetesimals). In both cases, one can therefore expect that the average water content of accreted solids (which does not necessary reflect the final water content of planets as discussed below) can vary depending on the exact formation path of the planets. What is, however, not clear is whether it is possible to end up with a water budget that shows no trend. Indeed, one would expect, from a smooth and ordered migration of planets, that the water content should monotonically increase as a function of period, as we see, for example, in the case of Galilean satellites. Breaking such a monotonic trend requires exchanging the place of some planets, which requires a strong level of dynamical rearrangement resulting from gravitational interactions. Whether such a rearrangement is possible, while at the same time ending with a flat and resonant system, remains to be investigated.

Another possibility is that the final water budget of planets is not the one of the accreted solids. Indeed, during their formation, planets accrete a gas envelope (which may be lost afterward). The interaction of the gas envelope, the protoplanetary disk, and the accreted solids can lead, under some circumstances, to the loss of some of the accreted solid material (Alibert 2017); however, see Brouwers et al. (2018). This process results from the advection of gas from the disk to the planetary atmosphere, and such a process could prevent accretion of water by forming planets. As the efficiency of advection depends on different parameters (including planetary mass, gas density in the disk, and distance to the star) in a nontrivial way, it is not clear whether such a process can destroy a preexisting water content gradient, but it is likely to increase the scatter in the final water content.

The second conclusion of our study is that the maximum water content is of the order to $30 \%$. Interestingly enough, this value is smaller than the assumed water content in solids beyond the ice line, but at the same time similar to the maximum water content in solar system bodies up to the terrestrial mass range (see Figure 14). In fact, Ormel et al. (2017) predict moderate water budgets $(\sim 10 \%)$ for the TRAPPIST-1 planets when planetary embryos form at the water snow line, migrate to the inner disk, and grow by dry pebbles until a critical planet mass $\left(\sim 0.7 M_{\oplus}\right)$ is reached that prohibits further pebble accretion. A reduction of water budget for planets that accreted outside of the snow line can include processes such as desiccation of volatile-rich planetesimals by short-lived radionuclides (Grimm \& McSween 1993; Lichtenberg et al. 2018), giant impacts between embryos (Genda \& Abe 2005), and heating of planets and planetesimals during accretion and collisions, which is expected to be more efficient around low-mass stars (Lissauer 2007).

In any case, the water budget of the TRAPPIST-1 planets is an important constraint that needs to be fully considered in formation models. As a consequence, better determinations (e.g., by improving the mass, radius, or compositional constraints on refractory elements or atmospheric composition) of this quantity are key to making future progress in the understanding of the formation of the system.

\section{Conclusions}

The TRAPPIST-1 planets do not follow a single mass-radius trend, but there is some scatter among the bulk densities of planets. Here we have quantified the origin of this scatter, which is mostly due to different amounts of water, but also to some extent the sizes of rocky interiors and the thicknesses of gas envelopes.

Our analysis characterizes the nature of TRAPPIST-1 planet interiors while accounting for all available and relevant data. These include the correlated planetary masses and radii and stellar irradiation. In addition, we have tested different abundance constraints: a stellar proxy based on stars of similar metallicities, as well as a proxy that is based on the densest and probably a purely rocky planet of the system, planet e. The latter abundance proxy is unique to multiplanetary systems (see Section 4.2.1). Furthermore, there are data specific to multiplanetary systems that have not been considered in previous studies: the interdependency of planet masses between different 


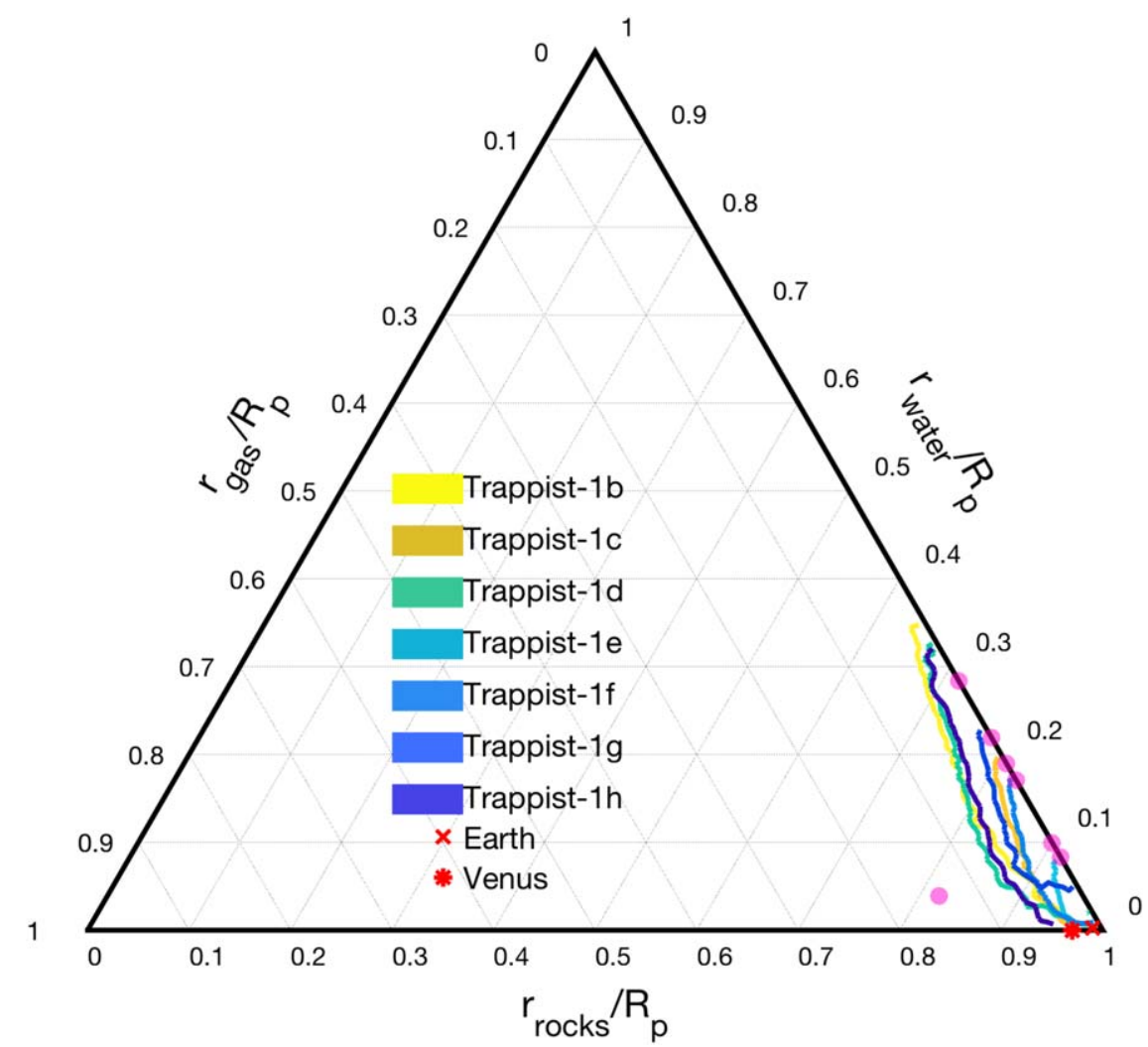

Figure 14. Ternary diagram showing the marginalized $2 \sigma$ distributions of rock-water-gas radius fractions for TRAPPIST-1 planets and scenario UCM. All three radius fractions sum up to 1 , which makes it possible to represent the distributions in a ternary diagram. For comparison, Earth and Venus are shown in red, and mean estimates for Europa, Ganymede, Callisto, Titan, Triton, Pluto, and Charon are shown in purple.

planets as derived from TTV analysis, and the interdependency of planet radii between different planets as derived from TTV analysis. Here we have developed a new resampling scheme (Section 3.4.3) that allowed us to incorporate the information on interdependent data (Section 4.2.2). The information that we can gain on the interiors by accounting for interdependent planetary data can be important (up to $20 \%$ differences), even as important as an improvement in mass and radius precision (Section 4.2.4).

We highlight that the precision on the differential planetary data is much better than on absolute masses and radii. This is because the latter includes stellar uncertainties, while the former does not. By accounting for the correlations among all seven masses, we formally use the knowledge on the differential masses. For multiplanetary systems, as demonstrated here for TRAPPIST-1, the use of differential planetary data is important for a thorough interior investigation.

Systematic biases of data can critically influence interior characterization. Care should be taken with our and all previous interior interpretations that critically depend on planetary masses and densities of TRAPPIST-1. Ongoing observational efforts indicate possible changes in mass accuracies within $\pm 24 \%$ (Demory 2018). In this case, all interiors could be consistent with an increasing water mass fraction with orbital period (within $2 \%-12 \%$ with respect to $1 \sigma$ errors) or a uniform water mass fraction of $7 \%$ (within $1 \sigma$ errors). This is in contrast with our findings based on the most recent data publications (Delrez et al. 2018; Grimm et al. 2018), which we summarize below:
1. TRAPPIST-1e can be a super-Mercury-type planet with non-Earth-like bulk abundance. This is obvious from the high bulk density as determined by Grimm et al. (2018) and was discussed by Suissa \& Kipping (2018). Here we have quantified the rocky composition of planet e to be characterized by $\mathrm{Fe} / \mathrm{Si}_{\mathrm{T} 1 \mathrm{e}}=11.2 \pm 5.7$ and $\mathrm{Mg} / \mathrm{Si}_{\mathrm{Tle}}=$ $5.7 \pm 3.7$. If the rocky composition of planet e were indeed different from the other planets, it could be due to a giant impact that has not only stripped off parts of the mantle (Benz et al. 1988) but also removed volatile-rich layers. Such a scenario would explain why planet e is much drier than the other planets. However, this scenario would require an impactor larger than planet e, which does not exist in the system, and it remains to be studied whether impacts are a reasonable scenario. In this scenario, the interiors predicted for all planets might be well described by $U$, with the exception of planet $e$, which is best described by $\mathrm{A}$.

2. Alternatively, TRAPPIST-1e may not be a superMercury-type planet. There are two interpretations possible under this premise:

i. First, it is possible that systematic errors of the TTV analysis due to the limited observation time and yet-undetected planets in the system may bias the planetary masses, including planet e. If this is the case, the stellar proxy would be an important constraint to favor rather Earth-like interiors for planet e, dismissing high bulk density interiors. Consequently, due to the interdependency of planetary data, the information kept in the level of incompatibility of data (in scenario 
$\mathrm{U}$ using the stellar proxy) propagates to all other planets, yielding better-constrained interiors by excluding some interior models. In other words, for all planets there is only a subset of interiors that is in agreement with all available data on all planets, their interdependencies, and the stellar proxy. If this case is true, all interiors are well described by scenario UCM.

ii. Second, it is possible that the interiors of TRAPPIST1 planets cannot be described by Earth-like interiors or the suggested stellar proxy. Instead the purely rocky interior of all planets is directly probed by the densest planet, planet e, assuming that all rocky interiors have similar ratios of rock-forming elements $(\mathrm{Mg}, \mathrm{Si}, \mathrm{Fe})$. In this case, all predicted interiors are best described by scenario $A$.

3. Differences between estimated interiors are large when comparing different abundance constraints ( $U$ and $A$ ). This demonstrates the need to better understand the relative amounts of refractory, rock-forming elements in dwarf systems, like TRAPPIST-1, which probably also depend on our knowledge of the age of the system. Unfortunately, direct estimates of the photosphere of the faint TRAPPIST-1 are unavailable. Possible abundance constraints based both on stars with similar metallicities (U) and on the densest planet (A) can be justified. However, it is difficult to state a clear preference.

4. The information that is kept in the interdependency of planetary data is a valuable constraint that can significantly affect interior estimates. For example, estimated median water budgets can vary up to $20 \%$ (compare scenarios U and UCM). Accounting for interdependency of planetary data compares with changes in data precision of $30 \%$.

5. Mass and radius data only carry limited information about planetary interiors, and additional data types are required to significantly improve interior estimates. For example, the improvement on predicted amounts of water due to more precise data ( $70 \%$ of nominal data uncertainties) is rather small compared to changes in abundance proxies ( $U$ and $A$ ).

6. Our inferred ranges of water contents of $0 \%-25 \%$ are high compared to terrestrial solar system planets and are smaller by a factor of two compared to predictions from formation studies (Alibert \& Benz 2016). Volatile-rich interiors of planets in dwarf star systems are predicted given that the water ice line is much closer to the star compared to a solar-like system.

7. There is no clear trend of volatile fraction with orbital period. This suggests that the accreted planetesimals were sufficiently mixed such as to blur otherwise expected increases of water fraction with distance from the star. A corresponding uniform water content is indeed possible within $2 \sigma$ error bars. Alternatively, migration may have rearranged the planets before they were captured in resonance.

8. Possible delivery of volatiles after formation by cometary impacts (Kral et al. 2018) of a few Earth oceans is tiny compared to our predicted water mass fractions. This means that the overall water budgets were accreted during formation. Also, the interior degeneracy is large such that uncertainties of predicted water masses are orders of magnitude larger than possible late-delivered amounts of volatiles. This implies that the data do not allow us to validate late delivery of volatiles.

9. The loss of volatiles as predicted by Bourrier et al. (2017) of several tens of Earth oceans is small compared to the total amount of water that shapes the planets, except for planet e. This implies that the ice mass fraction of the bulk accreted material does not significantly exceed $30 \%$. If data accuracy changes by up to $24 \%$, this upper limit could be significantly lower $(\sim 15 \%)$.

10. The uncertainty in our predicted water mass fractions stems from the degeneracy with the size, structure, and composition of the rocky interior, as well as with the characteristics of the overlying gas envelope. The estimated degeneracy will be generally lower if interior models are employed that only allow for limited variability, e.g., mantles of pure $\mathrm{MgSiO}_{3}$ as employed in Unterborn et al. (2017). Similarly, estimated degeneracies will be larger if interior models are used that allow for interiors that are unlikely to exist in nature, e.g., pure iron cores surrounded by gas envelopes as used in Suissa \& Kipping (2018).

Significant further improvements on our understanding of the TRAPPIST-1 planetary interiors are only expected with higher data accuracy and/or informative data other than those investigated here, which may include better understanding of their specific host star chemistry, spectroscopic constraints on atmospheric composition (e.g., with JWST, Ariel, E-ELT), or constraints on tidal parameters (e.g., Papaloizou et al. 2017).

With our study on TRAPPIST-1, we have explored the data types that are specific to multiplanetary systems. Such data will be relevant for the interior characterization of planets in other systems as well. First, there are correlations between the data of different planets that can carry crucial information for interior characterization. Second, we have demonstrated that it is possible to preferentially probe the rocky interiors of all planets by studying the densest planet of a multiplanetary system. This study provides new pathways for an improved interior characterization that is specific to multiplanetary systems.

This work was supported by the Swiss National Foundation under grant PZ00P2_174028. It was in part carried out within the frame of the National Centre for Competence in Research PlanetS.

\section{Appendix A Approximation of $\alpha_{\max }$}

There is a physical upper limit to the amount of warming by greenhouse gases. The Komabayashi-Ingersoll (KI) limit describes the maximum amount of radiation that can be transferred by a moist atmosphere, which occurs when the transparency $\tau_{\mathrm{s}}$ of the atmosphere becomes very small, i.e., $\tau_{\mathrm{s}}=\tau_{\text {limit }}$.

Here this limit is represented by $\alpha_{\max }$, which we roughly approximate as follows:

$$
\alpha_{\max }=\frac{T_{\text {limit }}}{T_{\text {star }} \sqrt{\frac{R_{\text {star }}}{2 a}}},
$$


where $R_{\text {star }}$ and $T_{\text {star }}$ are the radius and effective temperature of the host star, respectively, $a$ is the semimajor axis, and $T_{\text {limit }}$ is

$$
T_{\text {limit }}=\frac{T_{0}}{\ln \left(\frac{\kappa^{*} p_{0}}{\pi_{\text {imit } g}}\right)} .
$$

Here $T_{0}$ is the temperature at some vapor pressure $p_{0}$ (here we use $p_{0}=1 \times 10^{5} \mathrm{~Pa}$ and $T_{0}=373 \mathrm{~K}$ for water; Goldblatt \& Watson 2012), $\kappa$ and $\tau_{\text {limit }}$ are the opacity and optical depth at the KI limit, respectively, and $g$ is surface gravity. The fraction $\frac{\kappa}{\tau \text { init }}$ is approximated for Earth $\left(T_{\text {limit }} \approx 400 \mathrm{~K}\right)$ and is estimated to be $10^{-7}$ (in SI units). Thereby, $T_{\text {limit }}$ (Equation (20)) scales with the surface gravity. This is a rough estimate for $T_{\text {limit }}$ and thus $\alpha_{\max }$. More advanced modeling would be required to better determine this limit, but this is outside of the scope of this study.

Equation (20) is derived from $\tau_{\mathrm{s}}=\frac{\kappa^{*} p_{\mathrm{s}}}{g}$ and the ClausiusClapeyron equation, which relates the surface pressure $p_{\mathrm{s}}$ and temperature $T_{\mathrm{s}}$ :

$$
p_{\mathrm{s}}=p_{0} \exp \left(-\frac{T_{0}}{T_{\mathrm{s}}}\right)
$$

\section{Appendix B \\ Equivalence between Bayes's Theorem and the Tarantola- Valette Formulation}

The prior in the Valette formulation is described in data and model space $(\mathcal{D}$ and $\mathcal{M})$. If the data are a priori independent of the model, the prior can be written as

$$
\rho(\boldsymbol{d}, \boldsymbol{m})=\rho_{d}(\boldsymbol{d}) \rho_{m}(\boldsymbol{m}),
$$

where $\rho_{d}(\boldsymbol{d})$ and $\rho_{m}(\boldsymbol{m})$ are evaluated in data and model space, respectively. If the data-model mapping $g(\cdot)$ is exact, the forward density $\theta(\boldsymbol{d}, \boldsymbol{m})$ can be rewritten as

$$
\begin{aligned}
\theta(\boldsymbol{d}, \boldsymbol{m}) & =\theta(\boldsymbol{d} \mid \boldsymbol{m}) \theta(\boldsymbol{m}) \\
& =\theta(\boldsymbol{d} \mid \boldsymbol{m}) z(\boldsymbol{m}) \\
& =\delta(\boldsymbol{d}-g(\boldsymbol{m})) z(\boldsymbol{m}),
\end{aligned}
$$

where $\delta$ is the delta function. If the null information density $z$ $(\boldsymbol{d}, \boldsymbol{m})$ is constant, we can state the posterior as

$$
\begin{aligned}
p_{m}(\boldsymbol{m}) & =\int_{\mathcal{D}} p(\boldsymbol{d}, \boldsymbol{m}) d \boldsymbol{d} \\
& =\int_{\mathcal{D}} \frac{\rho_{d}(\boldsymbol{d}) \rho_{m}(\boldsymbol{m}) \delta(\boldsymbol{d}-g(\boldsymbol{m})) z(\boldsymbol{m})}{z(\boldsymbol{d}, \boldsymbol{m})} d \boldsymbol{d} \\
& =\rho_{d}(g(\boldsymbol{m})) p_{m}(\boldsymbol{m}) \\
& \equiv L_{d}(\boldsymbol{m}) p_{m}(\boldsymbol{m}),
\end{aligned}
$$

where $L_{d}(\boldsymbol{m})$ is the likelihood of the Bayes's formulation.

\section{ORCID iDs}

Simon L. Grimm (i) https://orcid.org/0000-0002-0632-4407

Yann Alibert (iD https://orcid.org/0000-0002-4644-8818

\section{References}

Alibert, Y. 2017, A\&A, 606, A69

Alibert, Y., \& Benz, W. 2017, A\&A, 598, L5

Barr, A. C., Dobos, V., \& Kiss, L. L. 2018, A\&A, 613, A37

Benz, W., Slattery, W. L., \& Cameron, A. 1988, Icar, 74, 516

Bolmont, E., Selsis, F., Owen, J. E., et al. 2016, MNRAS, 464, 3728

Bouchet, J., Mazevet, S., Morard, G., Guyot, F., \& Musella, R. 2013, PhRvB, 87, 094102

Bourrier, V., Ehrenreich, D., Wheatley, P. J., et al. 2017, A\&A, 599, L3

Brouwers, M., Vazan, A., \& Ormel, C. 2018, A\&A, 611, A65

Connolly, J. 2009, GGG, 10, Q10014

Crida, A., Ligi, R., Dorn, C., \& Lebreton, Y. 2018, ApJ, 860, 122

de Wit, J., Wakeford, H. R., Gillon, M., et al. 2016, Natur, 537, 69

de Wit, J., Wakeford, H. R., Lewis, N. K., et al. 2018, NatAs, 2, 214

Delrez, L., Gillon, M., Triaud, A., et al. 2018, MNRAS, 475, 3577

Demory, B. 2018, in TRAPPIST-1: The Sonata and Its Dominant 7th, Exoplanet II Meeting Program (Cambridge: Cambridge Univ. Press)

Dorn, C., Khan, A., Heng, K., et al. 2015, A\&A, 577, A83

Dorn, C., Venturini, J., Khan, A., et al. 2017, A\&A, 597, A37

Fortney, J. J., Marley, M. S., \& Barnes, J. W. 2007, ApJ, 659, 1661

Genda, H., \& Abe, Y. 2005, Natur, 433, 842

Gillon, M., Jehin, E., Lederer, S. M., et al. 2016, Natur, 533, 221

Gillon, M., Triaud, A. H., Demory, B.-O., et al. 2017, Natur, 542, 456

Ginzburg, S., Schlichting, H. E., \& Sari, R. 2016, ApJ, 825, 29

Goldblatt, C., \& Watson, A. J. 2012, RSPTA, 370, 4197

Grimm, R. E., \& McSween, H. Y., Jr 1993, Sci, 259, 653

Grimm, S. L., Demory, B.-O., Gillon, M., et al. 2018, A\&A, 613, A68

Kane, S. R. 2018, ApJL, 861, L21

Kral, Q., Wyatt, M. C., Triaud, A. H., et al. 2018, MNRAS, 479, 2649

Lichtenberg, T., Golabek, G. J., Burn, R., et al. 2018, EPSC, 12, 132

Lissauer, J. J. 2007, ApJL, 660, L149

Lodders, K. 2003, ApJ, 591, 1220

Lodders, K., Palme, H., \& Gail, H.-P. 2009, M\&PSA, 72, 5154

Luger, R., Sestovic, M., Kruse, E., et al. 2017, NatAs, 1, 0129

Marcus, R. A., Sasselov, D., Hernquist, L., \& Stewart, S. T. 2010, ApJL, 712, L73

Nimmo, F., \& Pappalardo, R. 2016, JGRE, 121, 1378

Ormel, C. W., Liu, B., \& Schoonenberg, D. 2017, A\&A, 604, A1

Papaloizou, J. C., Szuszkiewicz, E., \& Terquem, C. 2017, MNRAS, 476, 5032

Seager, S., Kuchner, M., Hier-Majumder, C., \& Militzer, B. 2007, ApJ, 669, 1279

Seager, S., \& Mallen-Ornelas, G. 2003, ApJ, 585, 1038

Sicardy, B., Ortiz, J., Assafin, M., et al. 2011, Natur, 478, 493

Sotin, C., Grasset, O., \& Mocquet, A. 2007, Icar, 191, 337

Stewart, S. T., \& Leinhardt, Z. M. 2009, ApJL, 691, L133

Suissa, G., \& Kipping, D. 2018, RNAAS, in press (arXiv:1804.10618)

Tarantola, A., \& Valette, B. 1982, RvGeo, 20, 219

Turbet, M., Bolmont, E., Leconte, J., et al. 2018, A\&A, 612, A86

Unterborn, C. T., Desch, S. J., Hinkel, N., \& Lorenzo, A. 2017, NatAs, in press (arXiv:1706.02689)

Unterborn, C. T., Hinkel, N. R., \& Desch, S. J. 2018, RNAAS, 2, 116

Vazan, A., Kovetz, A., Podolak, M., \& Helled, R. 2013, MNRAS, 434, 3283

Weiss, L. M., Rogers, L. A., Isaacson, H. T., et al. 2016, ApJ, 819, 83 